\title{
BILINEAR IDENTITIES INVOLVING THE $k$-PLANE TRANSFORM AND FOURIER EXTENSION OPERATORS
}

\author{
DAVID BELTRAN AND LUIS VEGA
}

\begin{abstract}
We prove certain $L^{2}\left(\mathbb{R}^{n}\right)$ bilinear estimates for Fourier extension operators associated to spheres and hyperboloids under the action of the $k$-plane transform. As the estimates are $L^{2}$-based, they follow from bilinear identities: in particular, these are the analogues of a known identity for paraboloids, and may be seen as higher-dimensional versions of the classical $L^{2}\left(\mathbb{R}^{2}\right)$-bilinear identity for Fourier extension operators associated to curves in $\mathbb{R}^{2}$.
\end{abstract}

\section{INTRODUCTION}

For $n \geqslant 2$, let $U$ be an open subset in $\mathbb{R}^{n-1}$ and $\phi: \mathbb{R}^{n-1} \rightarrow \mathbb{R}$ be a smooth function parametrising a hypersurface $S=\{(\xi, \phi(\xi)): \xi \in U\}$. Associated to $S$, define the Fourier extension operator

$$
E f(z):=\int_{U} e^{i(x \cdot \xi+t \phi(\xi))} f(\xi) \mathrm{d} \xi
$$

where $z=(x, t) \in \mathbb{R}^{n-1} \times \mathbb{R}$ and $f \in L^{1}(U)$. The terminology extension comes from the fact that $E$ is the adjoint operator to the restriction of the Fourier transform to $S$, that is $E^{*} h(\xi)=\widehat{h}(\xi, \phi(\xi))$. Stein observed in the late 1960s that under certain curvature hypothesis on $S$ it is possible to obtain $L^{p}(U)-L^{q}\left(\mathbb{R}^{n}\right)$ estimates for $E$ besides the trivial $L^{1}(U)-L^{\infty}\left(\mathbb{R}^{n}\right)$ estimate implied by Minkowski's inequality. In particular, the Fourier restriction conjecture asserts that if $S$ is compact and has everywhere non-vanishing Gaussian curvature

$$
\|E f\|_{L^{q\left(\mathbb{R}^{n}\right)}} \leqslant C\|f\|_{L^{p}(U)}
$$

should hold for all $q>\frac{2 n}{n-1}$ and $\frac{1}{q} \leqslant \frac{n-1}{n+1} \frac{1}{p^{\prime}}$. This conjecture is fully solved for $n=2[17,36]$, but is still open for $n \geqslant 3$ and constitutes one of the main open problems in Euclidean Harmonic Analysis. The first fundamental result in this direction was the Stein-Tomas [34, 31] restriction estimate

$$
\|E f\|_{L^{\frac{2(n+1)}{n-1}\left(\mathbb{R}^{n}\right)}} \leqslant C\|f\|_{L^{2}(U)}
$$

note that this estimate is best possible in terms of the exponent $q$ for $f \in L^{2}(U)$. Over the last few years, there has been a great interest in establishing the sharp value of $C$ and the existence and characterisation of extremisers in (1.1) depending on the underlying surface $S$ : see, for instance, [18] or the most recent survey [21].

Substantial improvements on (1.1) have been achieved over the last few decades. An important ingredient for this has been the bilinear and multilinear approach. Multilinear restriction estimates generally adopt the form

$$
\left\|\prod_{j=1}^{k} E_{j} f_{j}\right\|_{L^{q / k}\left(\mathbb{R}^{n}\right)} \leqslant C \prod_{j=1}^{k}\left\|f_{j}\right\|_{L^{p}\left(U_{j}\right)}
$$

Date: December 3, 2019.

2010 Mathematics Subject Classification. 42B37, 35A23.

Key words and phrases. $k$-plane transform; Fourier extension operators; bilinear identities.

The authors were supported by ERCEA Advanced Grant 2014669689 - HADE, by the MINECO project MTM2014-53850-P, by Basque Government project IT-641-13 and also by the Basque Government through the BERC 2018-2021 program and by Spanish Ministry of Economy and Competitiveness MINECO: BCAM Severo Ochoa excellence accreditation SEV-2017-0718. 
where the $E_{j}$ are associated to hypersurfaces $S_{j}$ satisfying certain transversality hypotheses. A key feature of these inequalities is that, under such additional hypotheses, it is possible to obtain estimates for $p=2$ and $\frac{2 n}{n-1}<q<\frac{2(n+1)}{n-1}$. The interested reader is referred, for instance, to $[35,32]$ for the theory of bilinear restriction estimates and to [4] for the multilinear approach; see also the survey papers $[33,1]$.

An elementary instance of a bilinear estimate is in fact the identity

$$
\left\|E_{1} f_{1} E_{2} f_{2}\right\|_{L^{2}\left(\mathbb{R}^{2}\right)}^{2}=(2 \pi)^{2} \int_{U_{1} \times U_{2}} \frac{\left|f_{1}\left(\xi_{1}\right)\right|^{2}\left|f_{2}\left(\xi_{2}\right)\right|^{2}}{\left|\phi_{1}^{\prime}\left(\xi_{1}\right)-\phi_{2}^{\prime}\left(\xi_{2}\right)\right|} \mathrm{d} \xi_{1} \mathrm{~d} \xi_{2},
$$

which follows from an application of Plancherel's theorem and a change of variables; note that under the transversality hypothesis $\left|\phi_{1}^{\prime}\left(\xi_{1}\right)-\phi_{2}^{\prime}\left(\xi_{2}\right)\right|>c>0$ for $\xi_{1} \in U_{1}, \xi_{2} \in U_{2}$, one may interpret the identity (1.3) in the framework of (1.2). Of course, the presence of $L^{2}$ on the left-hand side in (1.3) is key for the use of Plancherel's theorem. This bilinear approach has its roots in the work of Fefferman [17] and may also be extended to higher dimensions. Identifying $E_{j} f_{j}=\widehat{g_{j} \mathrm{~d} \mu_{j}},{ }^{1}$ where $g_{j}: \mathbb{R}^{n} \rightarrow \mathbb{R}$ is the lift of $f_{j}$ to $S_{j}$, i.e., $g_{j}\left(\xi, \phi_{j}(\xi)\right)=f_{j}(\xi)$ and $\mathrm{d} \mu_{j}$ is the parametrised measure in $S_{j}$ defined via

$$
\int_{\mathbb{R}^{n}} g(\eta) \mathrm{d} \mu(\eta)=\int_{U_{j}} g\left(\xi, \phi_{j}(\xi)\right) \mathrm{d} \xi
$$

one may obtain the $L^{2}\left(\mathbb{R}^{n}\right)$ bilinear estimate

$$
\left\|E_{1} f_{1} E_{2} f_{2}\right\|_{L^{2}\left(\mathbb{R}^{n}\right)}^{2} \leqslant\left\|\left|g_{1}\right|^{2} \mathrm{~d} \mu_{1} *\left|g_{2}\right|^{2} \mathrm{~d} \mu_{2}\right\|_{L^{1}\left(\mathbb{R}^{n}\right)}\left\|\mathrm{d} \mu_{1} * \mathrm{~d} \mu_{2}\right\|_{L^{\infty}\left(\mathbb{R}^{n}\right)} \leqslant C\left\|f_{1}\right\|_{L^{2}\left(U_{1}\right)}^{2}\left\|f_{2}\right\|_{L^{2}\left(U_{2}\right)}^{2}
$$

after an application of Plancherel's theorem and the Cauchy-Schwarz inequality, provided one assumes the transversality condition $\left\|\mathrm{d} \mu_{1} * \mathrm{~d} \mu_{2}\right\|_{L^{\infty}\left(\mathbb{R}^{n}\right)} \leqslant C<\infty$. It should be remarked that the Lebesgue exponent 2 on the left-hand side of (1.4) corresponds to $q=4 \geqslant \frac{2(n+1)}{n-1}$ if $n \geqslant 3$; note that in a bilinear formulation the Lebesgue exponent is interpreted as $q / 2$. This is very much in contrast to the setting described in (1.2), in which the main goal is to obtain estimates when $q<\frac{2(n+1)}{n-1}$; bilinear and multilinear estimates of that type are deep and difficult and will not be explored in this paper.

It is interesting to compare (1.3) and (1.4). The first observation is that (1.3) is an identity, whilst (1.4) is an inequality. The second is the presence of the weight factor $\left|\phi_{1}^{\prime}\left(\xi_{1}\right)-\phi_{2}^{\prime}\left(\xi_{2}\right)\right|^{-1}$ in (1.3); the transversality weight $\left|\mathrm{d} \mu_{1} * \mathrm{~d} \mu_{2}\right|$ in (1.4) does not necessarily have a closed form in terms of the variables of integration of $f_{1}$ and $f_{2}$.

The main purpose of this paper is to further exploit the elementary 2-dimensional analysis in (1.3) in a higher dimensional setting. More precisely, we wish to obtain a bilinear identity in higher dimensions which incorporates an explicit weight factor amounting to some transversality condition; we note that an alternative higher dimensional version of (1.3) has recently been obtained by Bennett and Iliopoulou [5] in a $n$-linear level. In our goal of obtaining bilinear identities, we shall replace the $L^{2}\left(\mathbb{R}^{n}\right)$ in (1.4) by a mixed-norm $L^{1}\left(\mathbb{R}^{n-2}\right) \times L^{2}\left(\mathbb{R}^{2}\right)$. Given $x=\left(\bar{x}, x^{\prime \prime}\right) \in \mathbb{R}^{n-2} \times \mathbb{R}^{2}$, taking the $L^{1}$-norm in the $\bar{x}$ variables will essentially reduce matters to a 2-dimensional analysis in the $x^{\prime \prime}=\left(x_{n-1}, x_{n}\right)$ variables, where the resulting extension operators $E_{1}$ and $E_{2}$ will correspond to sections of the original surfaces by 2 -dimensional planes parallel to $\xi_{1}=\cdots=\xi_{n-2}=0$. The existence of such bilinear identities has already been established by Planchon and the second author [29] if the underlying hypersurfaces are paraboloids. The motivation in their work came from the relevant role played by these types of inequalities in the global behaviour of large solutions of nonlinear Schrödinger equations; see the next subsection for further details. Here we further explore whether bilinear identities hold for two other fundamental surfaces: the sphere and the hyperboloid.

Before describing our results in detail we shall first review the known results in the case of paraboloids, as they will provide the framework and context to understand our results.

\footnotetext{
${ }^{1}$ See $\S 2.1$ for the non-standard normalisation chosen for the Fourier transform $\hat{\imath}$
} 
1.1. Estimates for paraboloids and connections to Schrödinger equations. In recent years, starting with the work of Ozawa and Tsutsumi [28] for the paraboloid $S_{1}=S_{2}=\left\{\left(\xi,|\xi|^{2}\right)\right.$ : $\left.\xi \in \mathbb{R}^{n-1}\right\}$, there has been an increasing interest in understanding the weight $\left|\mathrm{d} \mu_{1} * \mathrm{~d} \mu_{2}\right|$ in (1.4) so that a $L^{2}$-bilinear estimate

$$
\left\|E_{1} f_{1} E_{2} f_{2}\right\|_{L^{2}\left(\mathbb{R}^{n}\right)}^{2} \leqslant C \int_{U_{1} \times U_{2}} K_{S_{1}, S_{2}}\left(\xi_{1}, \xi_{2}\right)\left|f_{1}\left(\xi_{1}\right)\right|^{2}\left|f_{2}\left(\xi_{2}\right)\right|^{2} \mathrm{~d} \xi_{1} \mathrm{~d} \xi_{2}
$$

holds for some kernel $K_{S_{1}, S_{2}}$ and such that the constant $C$ is best possible; in many cases, extremisers for the above kinds of inequalities have also been characterised. This has been mostly studied for paraboloids [10], cones [7], spheres [20,11] and hyperboloids [27, 23], with the corresponding natural interpretations in PDE.

It should be noted that the bilinear estimates (1.3) and (1.4) also hold when $E_{2} f_{2}$ is replaced by its complex conjugate $\overline{E_{2} f_{2}}$. This is, of course, of interest when $S_{1}=S_{2}$ and $f_{1}=f_{2}$, as then the bilinear estimates can be reinterpreted as $L^{2}$ estimates of $|E f|^{2}$. In particular, in the case of paraboloids, the identity (1.3) may be reinterpreted as

$$
\left.\left.\int_{\mathbb{R} \times \mathbb{R}}\left|D_{x}\right| u\right|^{2}\right|^{2} \mathrm{~d} x \mathrm{~d} t=\frac{1}{2(2 \pi)^{2}} \int_{\mathbb{R} \times \mathbb{R}}|\xi-\eta|\left|\widehat{u_{0}}(\xi)\right|^{2}\left|\widehat{u_{0}}(\eta)\right|^{2} \mathrm{~d} \xi \mathrm{d} \eta
$$

or simply

$$
\left.\left.\int_{\mathbb{R} \times \mathbb{R}}\left|D_{x}^{1 / 2}\right| u\right|^{2}\right|^{2} \mathrm{~d} x \mathrm{~d} t=\frac{1}{2}\left\|u_{0}\right\|_{L^{2}(\mathbb{R})}^{2}\left\|u_{0}\right\|_{L^{2}(\mathbb{R})}^{2}
$$

in order to avoid the singularity of the resulting weight $\left|\phi^{\prime}(\xi)-\phi^{\prime}(\eta)\right|=2|\xi-\eta|$; here we interpret the extension operator $u(x, t)=E \widetilde{u_{0}}(x, t)$ as the solution of the free Schrödinger equation $i \partial_{t} u-\Delta u=0$ in $\mathbb{R}^{d}$ associated to the initial data $u(x, 0)=u_{0}(x)$, with the normalisation of the Fourier transform considered in $\$ 2.1$. Note that, for this specific case, it is crucial that the multiplier associated to $D_{x}$ coincides precisely with $\left|\phi^{\prime}(\xi)-\phi^{\prime}(\eta)\right|$. Moreover, Ozawa and Tsutsumi [28] made use of the Radon transform to obtain the higher dimensional version

$$
\left\|(-\Delta)^{(2-d) / 4}|u|^{2}\right\|_{L_{x, t}^{2}\left(\mathbb{R}^{d} \times \mathbb{R}\right)}^{2} \leqslant \mathbf{O T}(d)\left\|u_{0}\right\|_{L^{2}\left(\mathbb{R}^{d}\right)}^{2}\left\|u_{0}\right\|_{L^{2}\left(\mathbb{R}^{d}\right)}^{2}
$$

where the constant $\mathbf{O T}(d)=\frac{2^{-d} \pi^{(2-d) / 2}}{\Gamma(d / 2)}$ is sharp after verifying that for $u_{0}(x)=e^{-|x|^{2}}$ the inequality becomes an identity; see also $[10,3]$.

The interest of Ozawa and Tsutsumi comes from the noninear Schrödinger equation

$$
i \partial_{t} u+\partial^{2} u=i \lambda\left(\partial|u|^{2}\right) u+f(u)
$$

where $\lambda \in \mathbb{R}$ and $f$ is a nonlinear interaction, which can be taken to be zero for simplicity of this exposition. In [28] the authors proved a well-posedness result in the Sobolev space $H^{1 / 2}(\mathbb{R})$. This was a non-trivial task due to the presence of the derivative term $\partial|u|^{2}$ on the right-hand side of the equation (1.9). The advantage of (1.7) (or (1.8) when $d=1$ ) as opposed to an inequality of the type (1.5) is the gain in derivatives of the solution with respect to the intial data, which allowed the authors to treat the term $\partial|u|^{2}$ as a perturbation.

The result by Ozawa and Tsutsumi was not further explored until [29], where Planchon and the second author established certain higher dimensional analogues of the $\mathbb{R}^{1+1}$ identity (1.6). Their identities also involved the Radon transform in the spatial variables ${ }^{2}$, which in fact features in the statement. Recall that given a linear $k$-dimensional subspace $\pi \in \mathcal{G}_{k, n}$ and $y \in \pi^{\perp}$, the $k$-plane transform of a function $f$ belonging to a suitable a priori class is defined as

$$
T_{k, n} f(\pi, y):=\int_{\pi} f(x+y) \mathrm{d} \lambda_{\pi}(x)
$$

\footnotetext{
${ }^{2}$ Note that the Radon transform in the spatial variables in $u(x, t)$ amounts to a $(n-2)$-plane transform in the context of the extension operators $E f(z)$.
} 
where $\mathcal{G}_{k, n}$ denotes the Grassmanian manifold of all $k$-dimensional subspaces in $\mathbb{R}^{n}$ and $\mathrm{d} \lambda_{\pi}$ is the induced Lebesgue measure on $\pi$. The cases $k=1$ and $k=n-1$ correspond to the X-ray transform $X$ and the Radon transform ${ }^{3} \mathcal{R}$ respectively. With this notation, the following was shown in [29].

Theorem 1.1 ([29]). Let $n \geqslant 2$ and $\omega \in \mathbb{S}^{d-1}$. Then,

$$
\int_{\mathbb{R}} \int_{\mathbb{R}}\left|\partial_{s} \mathcal{R}\left(|u(\cdot, t)|^{2}\right)(\omega, s)\right|^{2} \mathrm{~d} s \mathrm{~d} t+J_{\omega}(u)=\frac{\pi}{(2 \pi)^{2 d+1}} \int_{\mathbb{R}^{d}} \int_{\mathbb{R}^{d}}\left|(\xi-\eta) \cdot \omega \| \widehat{u_{0}}(\xi)\right|^{2}\left|\widehat{u_{0}}(\eta)\right|^{2} \mathrm{~d} \xi \mathrm{d} \eta
$$

where

$\left.J_{\omega}(u):=\int_{\mathbb{R}} \int_{\mathbb{R}} \int_{(\langle\omega\rangle \perp)^{2}}\left|u(x+s \omega, t) \partial_{s} u(y+s \omega, t)-u(y+s \omega, t) \partial_{s} u(x+s \omega, t)\right|^{2} \mathrm{~d} \lambda_{(\langle\omega\rangle}\right)^{2}(x, y) \mathrm{d} s \mathrm{~d} t$.

Note that fixing $\omega=e_{d}$ (or any other coordinate vector) in (1.10) above, the first term on the left-hand side amounts to $\left.\left\|\partial_{s}\right\||u|^{2}\left\|_{L^{1}\left(\mathbb{R}^{d-1}\right)}\right\|_{L_{x_{d}, t}^{2}}^{2} \mathbb{R}^{2}\right)$, which in the absence of the derivative $\partial_{s}$ becomes $\|u\|_{L_{x_{d}, t}^{4}\left(\mathbb{R}^{2} ; L^{2}\left(\mathbb{R}^{d-1}\right)\right)}^{4}$; note the contrast with the $L^{4}$-nature of (1.3) and (1.4).

The approach used in [29] to establish Theorem 1.1 uses integration-by-parts arguments and extends to versions of (1.10) for nonlinear Schrödinger equations with nonlinearity of the type $\pm|u|^{p-1} u$, where $p \geqslant 1$. The motivation in [29] is similar to that of Ozawa and Tsutsumi, and comes, more precisely, from the breakthrough result by Colliander, Keel, Staffilani, Takaoka, and Tao in [16], who established global well-posedness of the critical defocusing $3 d$ nonlinear Schrödinger equation (NLS)

$$
i \partial_{t} u+\Delta u=u|u|^{4}
$$

in the energy space. This builds up on a previous result of Bourgain [9], who showed the wellposedness under the assumption of radial symmetry. Bourgain used an ad hoc modification of a well-known weighted estimate, typically referred to as Morawetz inequality, proved in [24]; see also [25]. The weights are of the type $|x|^{-1}$ and therefore not translation invariant, leading to the well-posedness only under the radial assumption. To overcome that obstacle, the authors in [16] established a bilinear Morawetz estimate that avoids the loss of the translation symmetry. Whilst their strategy was successful in dimension 3, the method has some obstructions when considering nonlinear Schrödinger equations in dimensions 1 and 2. The nonlinear versions of the identities (1.10) were then used in [29] to prove certain lower dimensional well-posedness results, also obtained independently by Colliander, Grillakis and Tzirakis [15] by different methods. It is remarked that (1.10) has further applications, such as well-posedness in $3 d$ for exterior domains, scattering of solutions (see also [26]) or recovering Bourgain's [8] bilinear refinement of the Strichartz estimate; the reader is referred to [29] for further details.

Unfortunately, the bilinear identities (1.10) (or more precisely, the integration-by-parts proof method) are extremely rigid and they rely on the fact that the Schrödinger equation is a system with a quadratic dispersion relation. However, the connections of these estimates with Strichartz inequalities suggest that similar identities should also be true for general dispersion relations. This is what we start to explore in this paper for the particular case of the Helmholtz and Klein-Gordon equations. Our approach completely relies on Fourier Analysis techniques, after noting that (1.10) can be obtained from applications of Plancherel's theorem, in the spirit of (1.3) and (1.4). Of course, such a proof method only applies to linear problems, and it is therefore more natural to understand our results in the context of the interaction of the $k$-plane transform and $|E f|^{2}$, where the Fourier extension operator $E$ is associated to spheres and hyperboloids; see also the recent paper [2] or the upcoming preprint [6] for further examples of this interaction. Despite the lack of nonlinear results, we expect that the identification of bilinear identities for the linear Helmholtz and Klein-Gordon equations presented in this article will provide some light to develop methods based on direct integration by parts, which would be more amenable to nonlinear counterparts. This will be explored somewhere else in the future.

\footnotetext{
${ }^{3}$ The Radon transform $\mathcal{R} f$ is identified with a function in $\mathbb{S}_{+}^{n-1} \times \mathbb{R}$ setting $\mathcal{R} f(\omega, s) \equiv \mathcal{R} f\left(\langle\omega\rangle^{\perp}, s \omega\right)$.
} 


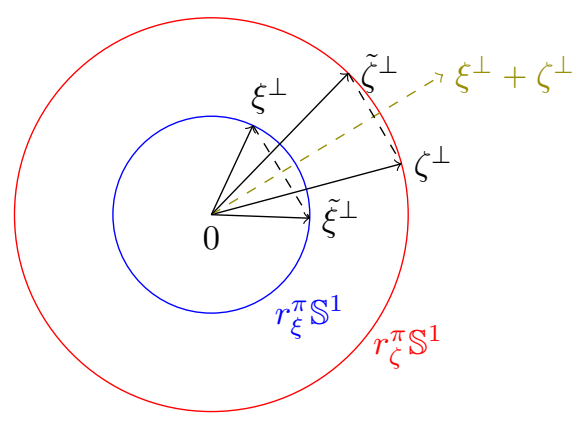

Figure 1. The new points $\tilde{\xi}^{\perp} \in r_{\xi}^{\pi} \mathbb{S}^{1}$ and $\tilde{\zeta}^{\perp} \in r_{\zeta}^{\pi} \mathbb{S}^{1}$ in $\pi^{\perp}$ are the reflected points of $\xi^{\perp}$ and $\zeta^{\perp}$ with respect to $\xi^{\perp}+\zeta^{\perp}$.

1.2. Estimates for the sphere. In the case of the sphere $\mathbb{S}_{r}^{n-1} \equiv r \mathbb{S}^{n-1}$ of radius $r$ in $\mathbb{R}^{n}$, consider the more classical form of the extension operator

$$
g \mapsto \widehat{g \mathrm{~d} \sigma_{r}^{n}}
$$

where $\mathrm{d} \sigma_{r}^{n}$ denotes the induced normalised Lebesgue measure on $\mathbb{S}_{r}^{n-1}$ and $g \in L^{1}\left(\mathbb{S}_{r}^{n-1}\right)$. The following $L^{2}$-identities for $T_{n-2, n}\left(\widehat{g_{1} \mathrm{~d} \sigma_{r}^{n}} \overline{\overline{g_{2} \mathrm{~d} \sigma_{r}^{n}}}\right)$ are obtained.

Theorem 1.2. Let $n \geqslant 3$. Let $\pi \in \mathcal{G}_{n-2, n}$ and let $\pi^{\perp}$ denote the orthogonal subspace to $\pi$. For each $z \in \mathbb{R}^{n}$, write $z=z^{\pi}+z^{\perp}$, where $z^{\pi}$ is the orthogonal projection of $z$ into $\pi$. Then

$$
\begin{aligned}
\int_{\pi^{\perp}}\left|\left(-\Delta_{y}\right)^{1 / 4} T_{n-2, n}\left(\widehat{g_{1} \mathrm{~d} \sigma_{r}^{n}} \overline{\overline{g_{2} \mathrm{~d} \sigma_{r}^{n}}}\right)(\pi, y)\right|^{2} \mathrm{~d} \lambda_{\pi^{\perp}}(y) \\
\quad=\mathbf{C}_{\mathbb{S}^{n-1}} \int_{\left(\mathbb{S}_{r}^{n-1}\right)^{2}} K_{\pi, \mathbb{S}_{r}^{n-1}}(\xi, \zeta) g_{1}(\xi) \bar{g}_{2}\left(\xi^{\pi}+\tilde{\xi}^{\perp}\right) g_{2}(\zeta) \bar{g}_{1}\left(\zeta^{\pi}+\tilde{\zeta}^{\perp}\right) \mathrm{d} \sigma_{r}^{n}(\xi) \mathrm{d} \sigma_{r}^{n}(\zeta)
\end{aligned}
$$

where

$$
K_{\pi, \mathbb{S}_{r}^{n-1}}(\xi, \zeta):=\frac{2}{\left|\xi^{\perp}+\zeta^{\perp}\right|}, \quad \mathbf{C}_{\mathbb{S}^{n-1}}:=(2 \pi)^{2(n-1)},
$$

$r_{\xi}=\sqrt{r^{2}-\left|\xi^{\pi}\right|^{2}}$ and $\tilde{\xi}^{\perp}, \tilde{\zeta}^{\perp} \in \pi^{\perp}$ are the reflected points of $\xi^{\perp}$ and $\zeta^{\perp}$ in $\pi^{\perp}$ with respect to the line passing through the origin and $\xi^{\perp}+\zeta^{\perp}$, that is $\xi^{\perp}+\zeta^{\perp}=\tilde{\xi}^{\perp}+\tilde{\zeta}^{\perp}$ (see Figure 1).

Of course, the $L^{2}$-nature of the inequality on its left-hand side allows one to take advantage of Plancherel's theorem. As briefly described before $\S 1.1$, the key presence of the $(n-2)$-plane transform reduces the problem to a 2-dimensional analysis, and one is left to understand the convolution of two weighted measures associated to concentric circles of different radii in the subspace $\pi^{\perp} \simeq \mathbb{R}^{2}$. The main advantage with respect to (1.4) is that in this setting it is possible to express $h_{1} \mathrm{~d} \sigma_{r_{1}}^{2} * h_{2} \mathrm{~d} \sigma_{r_{2}}^{2}\left(\xi^{\perp}+\zeta^{\perp}\right)$ as the weight $\mathrm{d} \sigma_{r_{1}}^{2} * \mathrm{~d} \sigma_{r_{2}}^{2}\left(\xi^{\perp}+\zeta^{\perp}\right)$ times an evaluation of the functions $h_{1}$ and $h_{2}$ at points depending on $\xi^{\perp}$ and $\zeta^{\perp}$.

Several interesting corollaries can be deduced from Theorem 1.2; their short proofs will be given in $\S 5$. Given complex numbers $a, b, c, d \in \mathbb{C}$, the well known identity

$$
a \bar{b} \bar{c} d=\frac{1}{2}\left(|a c|^{2}+|b d|^{2}-|a \bar{c}-b \bar{d}|^{2}\right)+i \operatorname{Im}(a \bar{b} \bar{c} d)
$$

may be used in Theorem 1.2 to replace the 4 -linear wave interaction

$$
g_{1}(\xi) \bar{g}_{2}\left(\xi^{\pi}+\tilde{\xi}^{\perp}\right) g_{2}(\zeta) \bar{g}_{1}\left(\zeta^{\pi}+\tilde{\zeta}^{\perp}\right)
$$

in (1.11) by an alternative expression involving $\left|g_{1}(\xi)\right|^{2}\left|g_{2}(\zeta)\right|^{2}$ and which is closer in spirit to (1.10). 
Corollary 1.3. Let $n \geqslant 3$ and $\pi \in \mathcal{G}_{n-2, n}$. Then

$$
\begin{aligned}
\int_{\pi^{\perp}} \mid\left(-\Delta_{y}\right)^{1 / 4} & \left.T_{n-2, n}\left(\widehat{g_{1} \mathrm{~d} \sigma_{r}^{n}} \overline{\overline{g_{2} \mathrm{~d} \sigma_{r}^{n}}}\right)(\pi, y)\right|^{2} \mathrm{~d} y \\
& =\mathbf{C}_{\mathbb{S}^{n-1}} \int_{\left(\mathbb{S}_{r}^{n-1}\right)^{2}} K_{\pi, \mathbb{S}_{r}^{n-1}}(\xi, \zeta)\left|g_{1}(\xi)\right|^{2}\left|g_{2}(\zeta)\right|^{2} \mathrm{~d} \sigma_{r}^{n}(\xi) \mathrm{d} \sigma_{r}^{n}(\zeta)-I_{\pi, \mathbb{S}_{r}^{n-1}}\left(g_{1}, g_{2}\right)
\end{aligned}
$$

where

$$
I_{\pi, \mathbb{S}_{r}^{n-1}}\left(g_{1}, g_{2}\right):=\frac{\mathbf{C}_{\mathbb{S}^{n-1}}}{2} \int_{\left(\mathbb{S}_{r}^{n-1}\right)^{2}} K_{\pi, \mathbb{S}_{r}^{n-1}}(\xi, \zeta)\left|g_{1}(\xi) g_{2}(\zeta)-g_{2}\left(\xi^{\pi}+\tilde{\xi}^{\perp}\right) g_{1}\left(\zeta^{\pi}+\tilde{\zeta}^{\perp}\right)\right|^{2} \mathrm{~d} \sigma_{r}^{n}(\xi) \mathrm{d} \sigma_{r}^{n}(\zeta)
$$

Of course, the term $I_{\pi, \mathbb{S}_{r}^{n-1}}\left(g_{1}, g_{2}\right) \geqslant 0$ and is identically zero if $g_{1}$ and $g_{2}$ are constant functions, so it may be dropped from (1.13) at the expense of losing the identity, leading to a sharp inequality which fits in the context of (1.5). Thus, the term $I_{\pi, \mathbb{S}_{r}^{n-1}}\left(g_{1}, g_{2}\right)$ may be interpreted as the distance of such a resulting inequality to become an identity. ${ }^{4}$

As the $k$-plane transform satisfies the Fourier transform relation

$$
\mathcal{F}_{y} T_{k, n} f(\pi, \xi)=\widehat{f}(\xi) \quad \text { for } \xi \in \pi^{\perp},
$$

one may easily obtain by means of Plancherel's theorem the relation

$$
\|f\|_{L^{2}\left(\mathbb{R}^{n}\right)}^{2}=\frac{(2 \pi)^{-k}}{\left|\mathcal{G}_{n-k-1, n-1}\right|}\left\|\left(-\Delta_{y}\right)^{k / 4} T_{k, n} f\right\|_{L^{2}\left(\mathcal{G}_{k, n}, L^{2}\left(\pi^{\perp}\right)\right)}^{2}
$$

see $\S 2$ for further details. Thus, on averaging Theorem 1.2 over all $\pi \in \mathcal{G}_{n-2, n}$ one has the following.

Corollary 1.4. Let $n \geqslant 3$. Then

$$
\left\|(-\Delta)^{\frac{3-n}{4}}\left(\widehat{g_{1} \mathrm{~d} \sigma_{r}^{n}} \overline{\widehat{g_{2} \mathrm{~d} \sigma_{r}^{n}}}\right)\right\|_{L^{2}\left(\mathbb{R}^{n}\right)}^{2} \leqslant(2 \pi)^{2-n} \mathbf{C}_{\mathbb{S}^{n-1}} \int_{\left(\mathbb{S}_{r}^{n-1}\right)^{2}} K_{\mathbb{S}_{r}^{n-1}}(\xi, \zeta)\left|g_{1}(\xi)\right|^{2}\left|g_{2}(\zeta)\right|^{2} \mathrm{~d} \sigma_{r}^{n}(\xi) \mathrm{d} \sigma_{r}^{n}(\zeta)
$$

where

$$
K_{\mathbb{S}_{r}^{n-1}}(\xi, \zeta):=\frac{1}{\left|\mathcal{G}_{1, n-1}\right|} \int_{\mathcal{G}_{n-2, n}} K_{\pi, \mathbb{S}_{r}^{n-1}}(\xi, \zeta) \mathrm{d} \mu_{\mathcal{G}}(\pi)
$$

In the particular case $n=3$ and after setting $g_{1}=g_{2}$, the right-hand side in Corollary 1.4 amounts to a bilinear quantity appearing in the work of Foschi [19] on the sharp constant in the Stein-Tomas inequality (1.1) for $\mathbb{S}^{2}$. Thus, appealing to his work, one can deduce the following.

Corollary 1.5 (Stein-Tomas [34], Foschi [19]).

$$
\left\|\widehat{g \mathrm{~d} \sigma^{3}}\right\|_{L^{4}\left(\mathbb{R}^{3}\right)} \leqslant 2 \pi\|g\|_{L^{2}\left(\mathbb{S}^{2}\right)} .
$$

Besides the value for the sharp constant, Foschi [19] also showed that the only real valued extremisers are constant functions; the existence of extremisers was previously verified in [13, 14].

Solution to the Helmholtz equation. Consider the Helmholtz equation $\Delta u+k^{2} u=0$ in $\mathbb{R}^{n}$. If $\sup _{R>0} \frac{1}{R} \int_{B_{R}}|u|^{2}<\infty$, then there exists $g \in L^{2}\left(\mathbb{S}_{k}^{n-1}\right)$ such that $u=g \widehat{\mathrm{d} \sigma_{\mathbb{S}_{k}^{n-1}}}$. Theorem 1.2 and the subsequent corollaries may be then interpreted in that context.

\footnotetext{
${ }^{4}$ The inequality resulting from dropping $I_{\pi, \mathbb{S}_{r}^{n-1}}\left(g_{1}, g_{2}\right)$ in (1.13) may be obtained more directly by an application of the Cauchy-Schwarz inequality: see $\$ 5.1$
} 


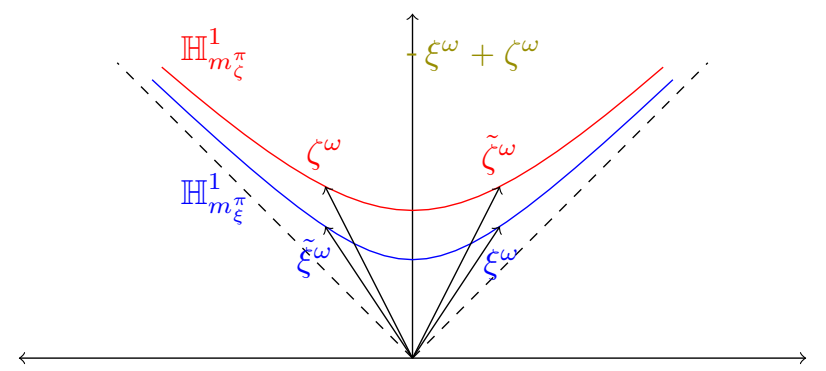

Figure 2. If $\xi^{\omega}+\zeta^{\omega}$ lies in the vertical axis, the new points $\tilde{\xi}^{\omega}$ and $\tilde{\zeta}^{\omega}$ are the reflected points of $\xi^{\omega}$ and $\zeta^{\omega}$ with respect to that axis. For ease of notation, $\xi^{\omega}$ is identified with the point $\left(\xi^{\omega}, \phi_{m_{\xi}^{\pi}}\left(\xi^{\omega}\right)\right) \in \mathbb{H}_{m_{\xi}^{\pi}}^{1}$, and similarly for the other points. Note that, in this situation, the Lorentz transformation $L$ in Theorem 1.6 is the identity. In general, the above situation results after applying $L$, which maps $\xi^{\omega}+\zeta^{\omega}$ to the vertical axis.

1.3. Estimates for the hyperboloid. A similar analysis to the one described for $\mathbb{S}^{n-1}$ may be carried for one of the components of the elliptic hyperboloid in $\mathbb{R}^{d+1}$, defined by

$$
\mathbb{H}_{m}^{d}:=\left\{\left(\xi, \xi_{d+1}\right) \in \mathbb{R}^{d} \times \mathbb{R}: \xi_{d+1}=\phi_{m}(\xi):=\sqrt{m^{2}+|\xi|^{2}}\right\}
$$

and equipped with the Lorentz invariant measure $\mathrm{d} \sigma_{\mathbb{H}_{m}^{d}}$ (see $\left.\S 2.3\right)$, defined by

$$
\int_{\mathbb{H}_{m}^{d}} g\left(\xi, \xi_{d+1}\right) \mathrm{d} \sigma_{\mathbb{H}_{m}^{d}}\left(\xi, \xi_{d+1}\right)=\int_{\mathbb{R}^{d}} g\left(\xi, \phi_{m}(\xi)\right) \frac{\mathrm{d} \xi}{\phi_{m}(\xi)} .
$$

A function $f \in L^{1}\left(\mathbb{R}^{d}\right)$ is identified with its lift $g$ to $\mathbb{H}_{m}^{d}$, given by $g\left(\xi, \phi_{m}(\xi)\right)=f(\xi)$, and note

$$
\widehat{g \mathrm{~d} \sigma_{\mathbb{H}_{m}^{d}}}(x, t)=\int_{\mathbb{R}^{d}} e^{i x \cdot \xi} e^{i t \sqrt{m^{2}+|\xi|^{2}}} f(\xi) \frac{\mathrm{d} \xi}{\sqrt{m^{2}+|\xi|^{2}}}
$$

where $(x, t) \in \mathbb{R}^{n}=\mathbb{R}^{d} \times \mathbb{R}$. A natural reason to split into a space-time domain is in view of the connection of $\widehat{g \mathrm{~d} \sigma_{\mathbb{H}_{m}^{d}}}$ with the Klein-Gordon propagator $e^{i t \sqrt{m^{2}-\Delta}} f$; this will be further discussed below. Thus, considering the Radon transform in the space variables - as in (1.10) and as opposed to Theorem 1.2, where no time role is given and therefore $(n-2)$-plane transform is taken - one obtains the following (see $\S 2.3$ for the definition of Lorentz transformation).

Theorem 1.6. Let $d \geqslant 2$. Let $\omega \in \mathbb{S}_{+}^{d-1}$ and let $\pi:=\langle\omega\rangle^{\perp} \in \mathcal{G}_{d-1, d}$ be the orthogonal subspace to $\langle\omega\rangle$. For each $x \in \mathbb{R}^{d}$, write $x=x^{\pi}+x^{\omega} \omega$, where $x^{\omega}=x \cdot \omega$. Then

$$
\begin{aligned}
\int_{\mathbb{R}} \int_{\mathbb{R}}\left|\partial_{s}^{1 / 2} \mathcal{R}\left(\widehat{g_{1} \widehat{\mathrm{d} \sigma_{\mathbb{H}_{m}^{d}}}}(\cdot, t) \widehat{\widehat{g_{2} \mathrm{~d} \sigma_{\mathbb{H}_{m}^{d}}}}(\cdot, t)\right)(\omega, s)\right|^{2} \mathrm{~d} s \mathrm{~d} t \\
=\mathbf{C}_{\mathbb{H}^{d}} \int_{\left(\mathbb{R}^{d}\right)^{2}} K_{\omega, \mathbb{H}_{m}^{d}}(\xi, \zeta) f_{1}(\xi) \bar{f}_{2}\left(\xi^{\pi}+\tilde{\xi}^{\omega} \omega\right) f_{2}(\zeta) \bar{f}_{1}\left(\zeta^{\pi}+\tilde{\zeta}^{\omega} \omega\right) \frac{\mathrm{d} \xi}{\phi_{m}(\xi)} \frac{\mathrm{d} \zeta}{\phi_{m}(\zeta)}
\end{aligned}
$$

where

$$
K_{\omega, \mathbb{H}_{m}^{d}}(\xi, \zeta):=\frac{\left|\xi^{\omega}-\tilde{\xi}^{\omega}\right|^{1 / 2}\left|\zeta^{\omega}-\tilde{\zeta}^{\omega}\right|^{1 / 2}}{\left|\xi^{\omega} \phi_{m}(\zeta)-\zeta^{\omega} \phi_{m}(\xi)\right|} \quad \text { and } \quad \mathbf{C}_{\mathbb{H}^{d}}=(2 \pi)^{2 d}
$$

Above, the points $\left(\tilde{\xi}^{\omega}, \phi_{m_{\xi}^{\pi}}\left(\tilde{\xi}^{\omega}\right)\right) \in \mathbb{H}_{m_{\xi}^{\pi}}^{1}$ and $\left(\tilde{\zeta}^{\omega}, \phi_{m_{\zeta}^{\pi}}\left(\tilde{\zeta}^{\omega}\right)\right) \in \mathbb{H}_{m_{\zeta}^{\pi}}^{1}$ are the image under $L^{-1}$ of the reflected points of $L\left(\left(\xi^{\omega}, \phi_{m_{\xi}^{\pi}}\left(\xi^{\omega}\right)\right)\right)$ and $L\left(\left(\zeta^{\omega}, \phi_{m_{\zeta}^{\pi}}\left(\zeta^{\omega}\right)\right)\right)$ in $\mathbb{R}^{2}$ with respect to the vertical axis respectively, where $L$ is the unique Lorentz transformation mapping $\left(\xi^{\omega}+\zeta^{\omega}, \phi_{m_{\xi}^{\pi}}\left(\xi^{\omega}\right)+\phi_{m_{\zeta}^{\pi}}\left(\zeta^{\omega}\right)\right)$ to the vertical axis and $m_{\xi}^{\pi}:=\sqrt{m^{2}+\left|\xi^{\pi}\right|^{2}}$ (see Figure 2).

As in the case of the sphere, the use of the Radon transform in $\mathbb{R}^{d}$ and Plancherel's theorem reduces the above estimate to explicitly understand $h_{1} \mathrm{~d} \sigma_{\mathbb{H}_{m_{1}}^{1}} * h_{2} \mathrm{~d} \sigma_{\mathbb{H}_{m_{2}}^{1}}\left(\xi^{\omega}+\zeta^{\omega}, \phi_{m_{1}}\left(\xi^{\omega}\right)+\phi_{m_{2}}\left(\zeta^{\omega}\right)\right)$. 


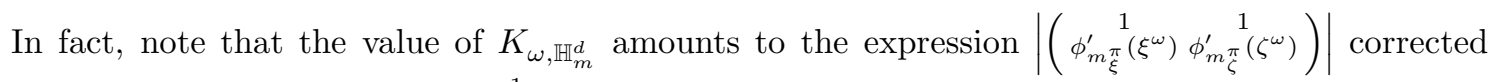

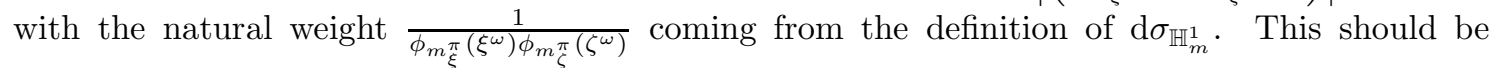
compared with the elementary two-dimensional identity (1.3). The presence of the numerator $\left|\xi^{\omega}-\tilde{\xi}^{\omega}\right|^{1 / 2}\left|\zeta^{\omega}-\tilde{\zeta}^{\omega}\right|^{1 / 2}$ is due to the action of $\partial_{s}^{1 / 2}$ on $\mathcal{R}\left(\widehat{g_{1} \mathrm{~d} \sigma_{\mathbb{H}_{m}^{d}}}(\cdot, t) \widehat{g_{2} \mathrm{~d} \sigma_{\mathbb{H}_{m}^{d}}}(\cdot, t)\right)$. Moreover, one can explicitly write $\tilde{\xi}^{\omega}$ and $\tilde{\zeta}^{\omega}$ in terms of $\xi, \zeta$ and $\omega$, leading to the more compact expression

$$
K_{\omega, \mathbb{H}_{m}^{d}}(\xi, \zeta)=\frac{2\left(\phi_{m}(\xi)+\phi_{m}(\zeta)\right)}{\left(\phi_{m}(\xi)+\phi_{m}(\zeta)\right)^{2}-((\xi+\zeta) \cdot \omega)^{2}}
$$

As in the case of the sphere, several corollaries can be deduced from Theorem 1.6. As for Corollary 1.3, one may use (1.12) to rewrite Theorem 1.6 in the spirit of Theorem 1.1.

Corollary 1.7. Let $d \geqslant 2$ and $\omega \in \mathbb{S}_{+}^{d-1}$. Then

$$
\begin{aligned}
\int_{\mathbb{R}} \int_{\mathbb{R}}\left|\partial_{s}^{1 / 2} \mathcal{R}\left(\widehat{g_{1} \mathrm{~d} \sigma_{\mathbb{H}_{m}^{d}}^{d}}(\cdot, t) \widehat{\widehat{g_{2} \mathrm{~d} \sigma_{\mathbb{H}_{m}^{d}}^{d}}}(\cdot, t)\right)(\omega, s)\right|^{2} \mathrm{~d} s \mathrm{~d} t \\
\quad=\mathbf{C}_{\mathbb{H}^{d}} \int_{\left(\mathbb{R}^{d}\right)^{2}} K_{\omega, \mathbb{H}_{m}^{d}}(\xi, \zeta)\left|f_{1}(\xi)\right|^{2}\left|f_{2}(\zeta)\right|^{2} \frac{\mathrm{d} \xi}{\phi_{m}(\xi)} \frac{\mathrm{d} \zeta}{\phi_{m}(\zeta)}-I_{\omega, \mathbb{H}_{m}^{d}}\left(f_{1}, f_{2}\right)
\end{aligned}
$$

where

$$
I_{\omega, \mathbb{H}_{m}^{d}}\left(f_{1}, f_{2}\right):=\frac{\mathbf{C}_{\mathbb{H}^{d}}}{2} \int_{\left(\mathbb{R}^{d}\right)^{2}} K_{\omega, \mathbb{H}_{m}^{d}}(\xi, \zeta)\left|f_{1}(\xi) f_{2}(\zeta)-f_{2}\left(\xi^{\pi}+\tilde{\xi}^{\omega} \omega\right) f_{1}\left(\zeta^{\pi}+\tilde{\zeta}^{\omega} \omega\right)\right|^{2} \frac{\mathrm{d} \xi}{\phi_{m}(\xi)} \frac{\mathrm{d} \zeta}{\phi_{m}(\zeta)}
$$

As $\mathcal{R}=T_{d-1, d}$, the use of the Plancherel's relation (1.15) after averaging over $\omega \in \mathbb{S}_{+}^{d-1}$ yields the following analogue of Corollary 1.4 for hyperboloids.

Corollary 1.8. Let $d \geqslant 2$. Then

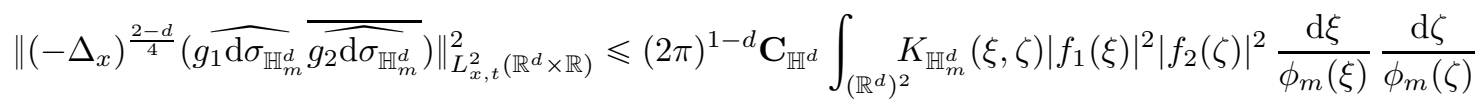

where

$$
K_{\mathbb{H}_{m}^{d}}(\xi, \zeta):=\frac{1}{2} \int_{\mathbb{S}^{d-1}} K_{\omega, \mathbb{H}_{m}^{d}}(\xi, \zeta) \mathrm{d} \sigma^{d}(\omega)
$$

The Klein-Gordon propagator. The solution to the Klein-Gordon equation $-\partial_{t}^{2} u+\Delta u=m^{2} u$ in $\mathbb{R}^{d} \times \mathbb{R}$, with initial data $u(x, 0)=f_{0}(x), \partial_{t} u(x, 0)=f_{1}(x)$ is given by

$$
u(x, t)=e^{i t \sqrt{m^{2}-\Delta}} f_{-}(x)+e^{-i t \sqrt{-\Delta}} f_{+}(x)
$$

where $f_{+}=\frac{1}{2}\left(f_{0}+i\left(\sqrt{m^{2}-\Delta}\right)^{-1} f_{1}\right)$ and $f_{-}=\frac{1}{2}\left(f_{0}-i\left(\sqrt{m^{2}-\Delta}\right)^{-1} f_{1}\right)$ and

$$
e^{ \pm i t \sqrt{m^{2}-\Delta}} f(x):=\frac{1}{(2 \pi)^{d}} \int_{\mathbb{R}^{d}} e^{i x \cdot \xi} e^{ \pm i t \sqrt{m^{2}+|\xi|^{2}}} \hat{f}(\xi) \mathrm{d} \xi
$$

Note that $e^{ \pm i t \sqrt{m^{2}-\Delta}} f(x)=(2 \pi)^{-d}\left(\widehat{g} \mathrm{~d} \sigma_{\mathbb{H}_{m}^{d}}\right)^{\wedge}(x, t)$; where $\widehat{g}$ is the lift of $\widehat{f} \sqrt{m^{2}+|\cdot|^{2}}$ to $\mathbb{H}_{m}^{d}$. Thus, Theorem 1.6 and Corollaries 1.7 and 1.8 may be re-interpreted in terms of $e^{i t \sqrt{m^{2}-\Delta}}$; in particular, setting $\mathbf{K G}(\mathbf{d})=(2 \pi)^{-4 d} \mathbf{C}_{\mathbb{H}^{d}}$, the estimate in Corollary 1.8 reads as

$$
\left\|\left(-\Delta_{x}\right)^{\frac{2-d}{4}}\left(e^{i t \sqrt{m^{2}-\Delta}} f_{1} \overline{e^{i t \sqrt{m^{2}-\Delta}} f_{2}}\right)\right\|_{L_{x, t}^{2}\left(\mathbb{R}^{d+1}\right)}^{2} \leqslant \mathbf{K G}(\mathbf{d}) \int_{\left(\mathbb{R}^{d}\right)^{2}} K_{\mathbb{H}_{m}^{d}}(\xi, \zeta)\left|\widehat{f}_{1}(\xi)\right|^{2}\left|\widehat{f}_{2}(\zeta)\right|^{2} \phi_{m}(\xi) \phi_{m}(\zeta) \mathrm{d} \xi \mathrm{d} \zeta
$$

Structure of the paper. Section 2 contains some notation and standard observations which will be useful throughout the paper. In Section 3 we revisit the convolution of weighted measures of circles and hyperbolas. Section 4 contains the proofs of Theorems 1.2 and 1.6 whilst Section 5 is concerned with the derivation of the several corollaries. Finally, we provide a Fourier analytic proof of Theorem 1.1 in Section 6, together with a further discussion on Fourier bilinear identities associated to paraboloids. 
Acknowledgements. The authors would like to thank Pedro Caro for several discussions at early stages of this project and Jon Bennett, Giuseppe Negro and Mateus Sousa for stimulating conversations. They also would like to thank the anonymous referee for a careful reading and many valuable suggestions.

\section{Notation AND PRELIMINARIES}

2.1. Fourier transform. We work with the normalisation of the Fourier transform

$$
\mathcal{F}(f)(\xi)=\widehat{f}(\xi)=\int_{\mathbb{R}^{n}} e^{i z \cdot \xi} f(z) \mathrm{d} z \quad \text { and } \quad \mathcal{F}^{-1}(f)(z)=\frac{1}{(2 \pi)^{n}} \int_{\mathbb{R}^{n}} e^{-i z \cdot \xi} f(\xi) \mathrm{d} \xi .
$$

With this normalisation,

$$
\widehat{f * g}=\hat{f} \cdot \widehat{g}, \quad \widehat{f g}(\xi)=(2 \pi)^{-n} \widehat{f} * \widehat{g}(\xi), \quad \widehat{\hat{f}}(z)=(2 \pi)^{n} \tilde{f}(z), \quad \widehat{\hat{\hat{f}}}(z)=(2 \pi)^{n} \bar{f}(z),
$$

where $\tilde{f}(z):=f(-z)$, Plancherel's theorem adopts the form

$$
\|\widehat{f}\|_{L^{2}\left(\mathbb{R}^{n}\right)}=(2 \pi)^{n / 2}\|f\|_{L^{2}\left(\mathbb{R}^{n}\right)} .
$$

The $n$-dimensional Dirac delta, denoted by $\delta_{n}$ is understood as

$$
\delta_{n}(a)=\frac{1}{(2 \pi)^{n}} \int_{\mathbb{R}^{n}} e^{i a \cdot z} \mathrm{~d} z .
$$

2.2. $k$-plane transform. The Grassmannian manifold $\mathcal{G}_{k, n}$ of all $k$-dimensional subspaces of $\mathbb{R}^{n}$ is equipped with an invariant measure $\mathrm{d} \mu_{\mathcal{G}}$ under the action of the orthogonal group. This measure is unique up to a constant, and is chosen to be normalised as

$$
\left|\mathcal{G}_{k, n}\right|=\int_{\mathcal{G}_{k, n}} \mathrm{~d} \mu_{\mathcal{G}}(\pi)=\frac{\left|\mathbb{S}^{n-1}\right| \cdots\left|\mathbb{S}^{n-k}\right|}{\left|\mathbb{S}^{k-1}\right| \cdots\left|\mathbb{S}^{0}\right|}
$$

Given $\pi \in \mathcal{G}_{k, n}$ and $\xi \in \pi^{\perp}$, the relation (1.14) between the $k$-plane transform $T_{k, n}$ and the Fourier transform easily follows from the definition

$$
\mathcal{F}_{y} T_{k, n} f(\pi, \xi)=\int_{\pi^{\perp}} e^{i y \cdot \xi} T_{k, n} f(\pi, y) \mathrm{d} \lambda_{\pi^{\perp}}(y)=\int_{\pi^{\perp}} e^{i y \cdot \xi} \int_{\pi} f(x+y) \mathrm{d} \lambda_{\pi}(x) \mathrm{d} \lambda_{\pi^{\perp}}(y)=\widehat{f}(\xi)
$$

after changing variables $z=x+y$ and noting that $\xi \cdot x=0$ for $\xi \in \pi^{\perp}$. This and the known identity (see, for instance, [22, Chapter 2])

$$
\int_{\mathbb{S}^{n-1}} f(\omega) \mathrm{d} \sigma^{n}(\omega)=\frac{1}{\left|\mathcal{G}_{n-k-1, n-1}\right|} \int_{\mathcal{G}_{k, n}} \int_{\mathbb{S}^{n-1} \cap \pi^{\perp}} f(\omega) \mathrm{d} \sigma_{\pi^{\perp}}^{n}(\omega) \mathrm{d} \mu_{\mathcal{G}}(\pi)
$$

yield via Plancherel's theorem and a change to polar coordinates the Plancherel-type identity (1.15) for the $k$-plane transform.

2.3. Lorentz transformations. The Lorentz group $\mathcal{L}$ is defined as the group of invertible linear transformations in $\mathbb{R}^{d+1}$ preserving the bilinear form

$$
(z, u) \mapsto z_{d+1} u_{d+1}-z_{d} u_{d}-\cdots-z_{1} u_{1} .
$$

It is well-known that the measure $\mathrm{d} \sigma_{\mathbb{H}_{m}^{d}}$ is invariant under the action of the subgroup of $\mathcal{L}$ that preserves the hyperboloid $\mathbb{H}_{m}^{d}$, denoted by $\mathcal{L}^{+}$. More precisely,

$$
\int_{\mathbb{H}_{m}^{d}} f \circ L \mathrm{~d} \sigma_{\mathbb{H}_{m}^{d}}=\int_{\mathbb{H}_{m}^{d}} f \mathrm{~d} \sigma_{\mathbb{H}_{m}^{d}}
$$

for all $L \in \mathcal{L}^{+}$. It is also a well-known fact that given $P=(\xi, \tau) \in \mathbb{R}^{d+1}$ with $\tau>|\xi|$, there exists a Lorentz transformation $L \in \mathcal{L}^{+}$such that $L(\xi, \tau)=\left(0, \sqrt{\tau^{2}-|\xi|^{2}}\right)$; see, for instance, [30]. For $d=1$, this transformation is given by

$$
L \equiv L_{\gamma_{P}}:=\left(\begin{array}{cc}
\cosh \gamma_{P} & -\sinh \gamma_{P} \\
-\sinh \gamma_{P} & \cosh \gamma_{P}
\end{array}\right), \quad \text { where } \quad \gamma_{P}:=\ln \sqrt{\frac{\tau+\xi}{\tau-\xi}}
$$




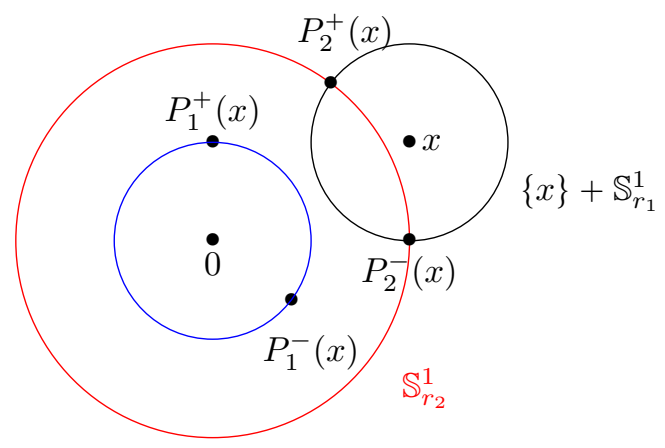

FiguRE 3 . The points $P_{2}^{+}(x), P_{2}^{-}(x) \in \mathbb{S}_{r_{2}}^{1} \cap\left(\{x\}+\mathbb{S}_{r_{1}}^{1}\right)$ and the points $P_{1}^{+}(x):=$ $x-P_{2}^{-}(x), P_{1}^{-}(x):=x-P_{2}^{+}(x) \in \mathbb{S}_{r_{1}}^{1}$. Note that $P_{1}^{+}(x), P_{1}^{-}(x) \in \mathbb{S}_{r_{1}}^{1} \cap\left(\{x\}+\mathbb{S}_{r_{2}}^{1}\right)$.

recall that $P$ may be expressed in hyperbolic coordinates as $P=(\xi, \tau)=\left(r_{P} \sinh \gamma_{P}, r_{P} \cosh \gamma_{P}\right)$, where $r_{P}:=\sqrt{\tau^{2}-\xi^{2}}$. The inverse Lorentz transformation that maps $\left(0, r_{P}\right)$ back to $(\xi, \tau)$ is given by $L_{-\gamma_{P}}$.

\section{Convolution of weighted measures}

As is discussed in the introduction, a key ingredient in the proofs of Theorems 1.2 and 1.6 is to understand convolutions of two weighted measures associated to concentric circles of different radii in $\mathbb{R}^{2}$ and to hyperbolas in $\mathbb{R}^{2}$ with the same perpendicular asymptotes and foci lying on the same line but with different major axis. The computation of such convolutions is standard; see, for instance, $[19,11]$ for the circular case or $[30,12]$ for the hyperbolic case. The main feature here is that the convolution is carried with respect to weighted measures, and, since the analysis is restricted to $\mathbb{R}^{2}$, one can give a precise evaluation of such weights at certain points.

3.1. Circles. Given $r \in \mathbb{R}_{+}$, let $\mathrm{d} \sigma_{r}^{2}$ denote the normalised Lebesgue measure of $\mathbb{S}_{r}^{1} \equiv r \mathbb{S}^{1}$, that is

$$
\int_{\mathbb{S}_{r}^{1}} g(\omega) \mathrm{d} \sigma_{r}^{2}(\omega)=\int_{\mathbb{S}^{1}} g(r \omega) \mathrm{d} \sigma^{2}(\omega)
$$

and recall that $\mathrm{d} \sigma^{2}(\omega)=\delta_{1}(1-|\omega|) \mathrm{d} \omega=2 \delta_{1}\left(1-|\omega|^{2}\right) \mathrm{d} \omega$, where $\mathrm{d} \omega$ denotes the Lebesgue measure on $\mathbb{R}^{2}$ and $\delta_{n}$ denotes the $n$-dimensional Dirac delta.

Given $0<r_{1} \leqslant r_{2}$, the domain of integration in $\mathrm{d} \sigma_{r_{1}}^{2} * \mathrm{~d} \sigma_{r_{2}}^{2}(x)$ is $\mathbb{S}_{r_{2}}^{1} \cap\left(\{x\}+\mathbb{S}_{r_{1}}^{1}\right)$. This set is non-empty if and only if $|x| \in\left[r_{2}-r_{1}, r_{2}+r_{1}\right]$ and consists of one point in the tangent case $|x|=r_{2}-r_{1}$ or $|x|=r_{2}+r_{1}$ and of two points otherwise. In the non-empty case, let $v_{x} \in \mathbb{S}^{1}$ denote the $\pi / 2$ degrees rotation of $x /|x|$ in the anti-clockwise direction, and let $P_{2}^{+}(x)$ and $P_{2}^{-}(x)$ denote the points in $\mathbb{S}_{r_{2}}^{1} \cap\left(\{x\}+\mathbb{S}_{r_{1}}^{1}\right)$ such that $P_{2}^{+}(x) \cdot v_{x} \geqslant 0$ and $P_{2}^{-}(x) \cdot v_{x} \leqslant 0$ respectively; note that $P_{2}^{+}(x)=P_{2}^{-}(x)$ in the tangent case. Define $P_{1}^{-}(x):=x-P_{2}^{+}(x) \in \mathbb{S}_{r_{1}}^{1}$ and $P_{1}^{+}(x):=x-P_{2}^{-}(x) \in \mathbb{S}_{r_{1}}^{1}$; note that $P_{1}^{+}(x), P_{1}^{-}(x) \in \mathbb{S}_{r_{1}}^{1} \cap\left(\{x\}+\mathbb{S}_{r_{2}}^{1}\right)$. Observe that $P_{j}^{+}(x)$ and $P_{j}^{-}(x)$ are reflected points one another with respect to the line passing through the origin containing $x$ : see Figure 3 .

Lemma 3.1. Let $r_{1}, r_{2} \in \mathbb{R}$ such that $0<r_{1} \leqslant r_{2}$. Then

$$
g_{1} \mathrm{~d} \sigma_{r_{1}}^{2} * g_{2} \mathrm{~d} \sigma_{r_{2}}^{2}(x)=\frac{2 g_{1}\left(P_{1}^{+}(x)\right) g_{2}\left(P_{2}^{-}(x)\right)+2 g_{1}\left(P_{1}^{-}(x)\right) g_{2}\left(P_{2}^{+}(x)\right)}{\sqrt{-\left(|x|^{2}-\left(r_{2}+r_{1}\right)^{2}\right)\left(|x|^{2}-\left(r_{2}-r_{1}\right)^{2}\right)}}
$$

if $|x| \in\left[r_{2}-r_{1}, r_{2}+r_{1}\right]$. 


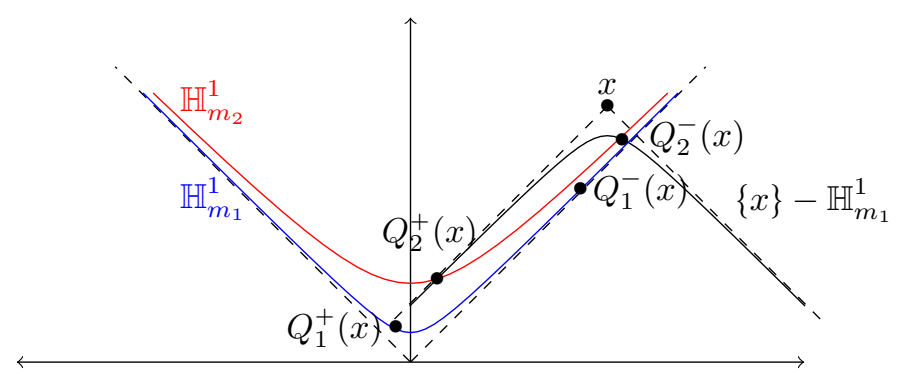

FiguRE 4. The points $Q_{2}^{+}(x), Q_{2}^{-}(x) \in \mathbb{H}_{m_{2}}^{1} \cap\left(\{x\}-\mathbb{H}_{m_{1}}^{1}\right)$ and the points $Q_{1}^{+}(x):=$ $x-Q_{2}^{-}(x), Q_{1}^{-}(x):=x-Q_{1}^{+}(x) \in \mathbb{H}_{m_{1}}^{1}$.

Proof. A standard computation shows (see for instance [19, Lemma 2.2] or [11, Lemma 5] for similar arguments)

$$
\begin{aligned}
g_{1} \mathrm{~d} \sigma_{r_{1}}^{2} * g_{2} \mathrm{~d} \sigma_{r_{2}}^{2}(x) & =\int_{\mathbb{S}^{1}} \int_{\mathbb{S}^{1}} g_{1}\left(r_{1} \omega_{1}\right) g_{2}\left(r_{2} \omega_{2}\right) \delta_{2}\left(x-r_{1} \omega_{1}-r_{2} \omega_{2}\right) \mathrm{d} \sigma^{2}\left(\omega_{1}\right) \mathrm{d} \sigma^{2}\left(\omega_{2}\right) \\
& =\frac{2}{r_{2}^{2}} \int_{\mathbb{S}^{1}} \int_{\mathbb{R}^{2}} g_{1}\left(r_{1} \omega_{1}\right) g_{2}\left(r_{2} \omega_{2}\right) \delta_{2}\left(\frac{x}{r_{2}}-\frac{r_{1}}{r_{2}} \omega_{1}-\omega_{2}\right) \delta_{1}\left(1-\left|\omega_{2}\right|^{2}\right) \mathrm{d} \sigma^{2}\left(\omega_{1}\right) \mathrm{d} \omega_{2} \\
& =\frac{1}{r_{1}|x|} \int_{\mathbb{S}^{1}} g_{1}\left(r_{1} \omega_{1}\right) g_{2}\left(x-r_{1} \omega_{1}\right) \delta_{1}\left(\frac{r_{2}^{2}}{2 r_{1}|x|}-\frac{|x|}{2 r_{1}}-\frac{r_{1}}{2|x|}+\frac{x}{|x|} \cdot \omega_{1}\right) \mathrm{d} \sigma^{2}\left(\omega_{1}\right) \\
& =: \mathrm{I}^{+}(x)+\mathrm{I}^{-}(x)
\end{aligned}
$$

where $\mathrm{I}^{+}(x)$ corresponds to the integration over $\mathbb{S}_{+}^{1}(x):=\left\{\omega \in \mathbb{S}^{1}: x \cdot \omega \geqslant 0\right\}$ and $\mathrm{I}^{-}(x)$ to the integration over $\mathbb{S}_{-}^{1}(x):=\mathbb{S}^{1} \backslash \mathbb{S}_{+}^{1}(x)=\left\{\omega \in \mathbb{S}^{1}: x \cdot \omega \leqslant 0\right\}$.

Denoting by $\alpha_{x}$ the clockwise angle between $e_{1}$ and $x$ and $P_{x}(u)=\left(\cos \left(\alpha_{x}+\arccos (u)\right), \sin \left(\alpha_{x}+\right.\right.$ $\arccos (u)))$, the expression for $\mathrm{I}^{+}(x)$ becomes, after a change of variable,

$$
\begin{aligned}
\mathrm{I}^{+}(x) & =\frac{1}{r_{1}|x|} \int_{-1}^{1} \delta_{1}\left(\frac{r_{2}^{2}}{2 r_{1}|x|}-\frac{|x|}{2 r_{1}}-\frac{r_{1}}{2|x|}+u\right)\left(1-u^{2}\right)^{-1 / 2} g_{1}\left(r_{1} P_{x}(u)\right) g_{2}\left(x-r_{1} P_{x}(u)\right) \mathrm{d} u \\
& =\frac{1}{r_{1}|x|}\left(1-\left(\frac{|x|^{2}+r_{1}^{2}-r_{2}^{2}}{2 r_{1}|x|}\right)^{2}\right)^{-1 / 2} g_{1}\left(P_{1}^{+}(x)\right) g_{2}\left(P_{2}^{-}(x)\right) \chi_{\left\{r_{2}-r_{1} \leqslant|x| \leqslant r_{2}+r_{1}\right\}}(x) \\
& =\frac{2 g_{1}\left(P_{1}^{+}(x)\right) g_{2}\left(P_{2}^{-}(x)\right)}{\sqrt{-\left(|x|^{2}-\left(r_{2}+r_{1}\right)^{2}\right)\left(|x|^{2}-\left(r_{2}-r_{1}\right)^{2}\right)}} \chi_{\left\{r_{2}-r_{1} \leqslant|x| \leqslant r_{2}+r_{1}\right\}}(x)
\end{aligned}
$$

noting that $r_{1} P_{x}(u)=P_{1}^{+}(x)$ after integrating in $u$. Indeed, if $y \in \mathbb{S}_{r_{1}}^{1} \cap\left(\{x\}+\mathbb{S}_{r_{2}}^{1}\right)$ one has $|y-x|^{2}=r_{2}^{2}$ and $|y|^{2}=r_{1}^{2}$. Therefore $\frac{y}{|y|} \cdot \frac{x}{|x|}=\frac{|x|^{2}+r_{1}^{2}-r_{2}^{2}}{2 r_{1}|x|}=u$, and $r_{1} P_{x}(u)=P_{1}^{+}(x)$ follows from noting that $P_{1}^{+}(x) \in \mathbb{S}_{r_{1}}^{1} \cap\left(\{x\}+\mathbb{S}_{r_{2}}^{1}\right)$ and $P_{1}^{+}(x) \cdot v_{x} \geqslant 0$. Arguing similarly for $\mathrm{I}^{-}(x)$ concludes the proof.

3.2. Hyperbolas. Consider the Lorentz invariant measure $\mathrm{d} \sigma_{\mathbb{H}_{m}^{1}}$ defined in (1.17). Given $0<$ $m_{1} \leqslant m_{2}$, the domain of integration in $\mathrm{d} \sigma_{\mathbb{H}_{m_{1}}^{1}} * \mathrm{~d} \sigma_{\mathbb{H}_{m_{2}}^{1}}(x)$ is $\mathbb{H}_{m_{2}}^{1} \cap\left(\{x\}-\mathbb{H}_{m_{1}}^{1}\right)$. Reasoning as in the previous case, this set is non-empty if and only if $\sqrt{x_{2}^{2}-x_{1}^{2}} \geqslant m_{1}+m_{2}$ and consists of one single point in the tangent case $\sqrt{x_{2}^{2}-x_{1}^{2}}=m_{1}+m_{2}$ and of two points otherwise; here $x=\left(x_{1}, x_{2}\right) \in \mathbb{R}^{2}$. In the non-empty case, let $Q_{2}^{+}(x)$ and $Q_{2}^{-}(x)$ denote the points in $\mathbb{H}_{m_{2}}^{1} \cap\left(\{x\}-\mathbb{H}_{m_{1}}^{1}\right)$ such that $\left(Q_{2}^{+}(x)-x\right) \cdot e_{1} \geqslant 0$ and $\left(Q_{2}^{-}(x)-x\right) \cdot e_{1} \leqslant 0$ respectively; of course, $Q_{2}^{+}(x)=Q_{2}^{-}(x)$ in the tangent case. Define $Q_{1}^{+}(x)=x-Q_{2}^{-}(x) \in \mathbb{H}_{m_{1}}^{1}$ and $Q_{1}^{-}(x)=x-Q_{2}^{+}(x) \in \mathbb{H}_{m_{1}}^{1}$ (see Figure 4 ). 
Lemma 3.2. Let $m_{1}, m_{2} \in \mathbb{R}$ such that $0<m_{1} \leqslant m_{2}$. For each $x=\left(x_{1}, x_{2}\right) \in \mathbb{R}^{2}$ such that $x_{2}^{2} \geqslant x_{1}^{2}$ one has

$g_{1} \mathrm{~d}{\sigma_{\mathbb{H}_{m_{1}}^{1}}} * g_{2} \mathrm{~d} \sigma_{\mathbb{H}_{m_{2}}^{1}}(x)=\frac{2 g_{1}\left(Q_{1}^{+}(x)\right) g_{2}\left(Q_{2}^{-}(x)\right)+2 g_{1}\left(Q_{1}^{-}(x)\right) g_{2}\left(Q_{2}^{+}(x)\right)}{\sqrt{\left(x_{2}^{2}-x_{1}^{2}\right)^{2}-2\left(x_{2}^{2}-x_{1}^{2}\right)\left(m_{1}^{2}+m_{2}^{2}\right)+\left(m_{1}^{2}-m_{2}^{2}\right)^{2}}} \chi_{\left\{\sqrt{x_{2}^{2}-x_{1}^{2}} \geqslant m_{1}+m_{2}\right\}}(x)$.

Proof. By invariance of the measure $\mathrm{d} \sigma_{\mathbb{H}^{1}}$ under Lorentz transformations, it suffices to prove the above identity for $x=(0, z)$. Indeed, note that if $L_{x} \in \mathcal{L}^{+}$is the Lorentz transformation satisfying $L_{x}(x)=(0, z)=\left(0, \sqrt{x_{2}^{2}-x_{1}^{2}}\right)$, then

$$
\begin{aligned}
g_{1} \mathrm{~d} \sigma_{\mathbb{H}_{m_{1}}^{1}} * g_{2} \mathrm{~d} \sigma_{\mathbb{H}_{m_{2}}^{1}}(x) & =\int_{\mathbb{H}_{m_{1}}^{1}} \int_{\mathbb{H}_{m_{2}}^{1}} g_{1}(\omega) g_{2}(\nu) \delta_{2}(x-\omega-\nu) \mathrm{d} \sigma_{\mathbb{H}_{m_{1}}^{1}}(\omega) \mathrm{d} \sigma_{\mathbb{H}_{m_{2}}^{1}}(\nu) \\
& =\int_{\mathbb{H}_{m_{1}}^{1}} \int_{\mathbb{H}_{m_{2}}^{1}} g_{1}\left(L_{x}^{-1}(\omega)\right) g_{2}\left(L_{x}^{-1}(\nu)\right) \delta_{2}((0, z)-\omega-\nu) \mathrm{d} \sigma_{\mathbb{H}_{m_{1}}^{1}}(\omega) \mathrm{d} \sigma_{\mathbb{H}_{m_{2}}^{1}}(\nu) \\
& =h_{1} \mathrm{~d} \sigma_{\mathbb{H}_{m_{1}}^{1}}^{1} * h_{2} \mathrm{~d} \sigma_{\mathbb{H}_{m_{2}}^{1}}(0, z)
\end{aligned}
$$

where $h_{j}=g_{j} \circ L_{x}^{-1}$; the reduction to the vertical axis then follows from noting that $h_{j}\left(Q_{\ell}^{ \pm}(0, z)\right)=$ $g_{j}\left(Q_{\ell}^{ \pm}(x)\right)$ for $j, \ell=1,2$. Next,

$$
\begin{aligned}
h_{1} \mathrm{~d} \sigma_{\mathbb{H}_{m_{1}}^{1}} * h_{2} \mathrm{~d} \sigma_{\mathbb{H}_{m_{2}}^{1}}(0, z) & =\int_{\mathbb{H}_{m_{1}}^{1}} \int_{\mathbb{H}_{m_{2}}^{1}} h_{1}(\omega) h_{2}(\nu) \delta_{2}\left((0, z)-\left(\omega_{1}, \omega_{2}\right)-\left(\nu_{1}, \nu_{2}\right)\right) \mathrm{d} \sigma_{\mathbb{H}_{m_{1}}^{1}}(\omega) \mathrm{d} \sigma_{\mathbb{H}_{m_{2}}^{1}}(\nu) \\
& =\int_{\mathbb{R}} h_{1}\left(\omega_{1}, \phi_{m_{1}}\left(\omega_{1}\right)\right) h_{2}\left(-\omega_{1}, \phi_{m_{2}}\left(\omega_{1}\right)\right) \frac{\delta_{1}\left(z-\phi_{m_{1}}\left(\omega_{1}\right)-\phi_{m_{2}}\left(\omega_{1}\right)\right)}{\phi_{m_{1}}\left(\omega_{1}\right) \phi_{m_{2}}\left(\omega_{1}\right)} \mathrm{d} \omega_{1}
\end{aligned}
$$

Splitting $\mathbb{R}=\mathbb{R}_{-} \cup \mathbb{R}_{+}$and doing the change of variables

$$
v=\phi_{m_{1}}\left(\omega_{1}\right)+\phi_{m_{2}}\left(\omega_{1}\right), \quad \text { with } \frac{\mathrm{d} \omega_{1}}{\phi_{m_{1}}\left(\omega_{1}\right) \phi_{m_{2}}\left(\omega_{1}\right)}=\frac{\mathrm{d} v}{\omega_{1} v},
$$

on each half-line one has

$$
\begin{aligned}
h_{1} \mathrm{~d} \sigma_{\mathbb{H}_{m_{1}}^{1}} * h_{2} \mathrm{~d} \sigma_{\mathbb{H}_{m_{2}}^{1}}(0, z)=\int_{m_{1}+m_{2}}^{\infty}\left(h_{1}\left(\omega_{1}, \phi_{m_{1}}\left(\omega_{1}\right)\right) h_{2}\left(-\omega_{1}, \phi_{m_{2}}\left(\omega_{1}\right)\right)\right. & \\
& \left.+h_{1}\left(-\omega_{1}, \phi_{m_{1}}\left(\omega_{1}\right)\right) h_{2}\left(\omega_{1}, \phi_{m_{2}}\left(\omega_{1}\right)\right)\right) \delta_{1}(z-v) \frac{\mathrm{d} v}{\omega_{1} v}
\end{aligned}
$$

where $\omega_{1}$ above is the function of $v$

$$
\omega_{1}=\omega_{1}(v):=\frac{\sqrt{v^{4}-2 v^{2}\left(m_{1}^{2}+m_{2}^{2}\right)+\left(m_{1}^{2}-m_{2}^{2}\right)^{2}}}{2 v} .
$$

Noting that $Q_{1}^{ \pm}(0, v)=\left( \pm \omega_{1}(v), \phi_{m_{1}}\left(\omega_{1}(v)\right)\right)$ and $Q_{2}^{ \pm}(0, v)=\left(\mp \omega_{1}(v), \phi_{m_{2}}\left(\omega_{1}(v)\right)\right)$, one has

$$
h_{1} \mathrm{~d} \sigma_{\mathbb{H}_{m_{1}}^{1}} * h_{2} \mathrm{~d} \sigma_{\mathbb{H}_{m_{2}}^{1}}(0, z)=\frac{2 h_{1}\left(Q_{1}^{+}(0, z)\right) h_{2}\left(Q_{2}^{-}(0, z)\right)+2 h_{1}\left(Q_{1}^{-}(0, z)\right) h_{2}\left(Q_{2}^{+}(0, z)\right)}{\sqrt{z^{4}-2 z^{2}\left(m_{1}^{2}+m_{2}^{2}\right)+\left(m_{1}^{2}-m_{2}^{2}\right)^{2}}} 1_{\left\{z \geqslant m_{1}+m_{2}\right\}},
$$

completing the proof.

\section{The proofs of Theorems 1.2 And 1.6}

4.1. Proof of Theorem 1.2. For simplicity we work on the unit sphere $r=1$; the result for $\mathbb{S}_{r}^{n-1}$ follows analogously. Given $\pi \in \mathcal{G}_{n-2, n}$, let $\pi^{\perp}$ denote its orthogonal subspace. For each $\xi \in \mathbb{R}^{n}$, write $\xi=\xi^{\pi}+\xi^{\perp}$, where $\xi^{\pi} \in \pi$ and $\xi^{\perp} \in \pi^{\perp}$, and let $r_{\xi}^{\pi}:=\sqrt{1-\left|\xi^{\pi}\right|^{2}}$. Given $x \in \pi$ and $y \in \pi^{\perp}$,

$$
\begin{aligned}
\widehat{g_{j} \mathrm{~d} \sigma^{n}}(x+y) & =\int_{\mathbb{S}^{n-1}} e^{i(x+y) \cdot \xi} g_{j}(\xi) \mathrm{d} \sigma^{n}(\xi) \\
& =\int_{\left|\xi^{\pi}\right| \leqslant 1} e^{i x \cdot \xi^{\pi}} \int_{r_{\xi}^{\pi} \mathbb{S}^{1}} e^{i y \cdot \xi^{\perp}} g_{j}\left(\xi^{\pi}+\xi^{\perp}\right) \mathrm{d} \sigma_{r_{\xi}^{\pi}}^{\perp}\left(\xi^{\perp}\right) \mathrm{d} \lambda_{\pi}\left(\xi^{\pi}\right) \\
& =\int_{\left|\xi^{\pi}\right| \leqslant 1} e^{i x \cdot \xi^{\pi}} \mathcal{F}^{\perp}\left(g_{j, \xi^{\pi}} \mathrm{d} \sigma_{r_{\xi}^{\pi}}^{\perp}\right)(y) \mathrm{d} \lambda_{\pi}\left(\xi^{\pi}\right)
\end{aligned}
$$


where $g_{j, \xi^{\pi}}(\omega):=g_{j}\left(\xi^{\pi}+\omega\right), \mathcal{F}^{\perp}$ denotes the Fourier transform in $\pi^{\perp}$ and $\mathrm{d} \sigma_{r_{\xi}^{\pi}}^{\perp}$ denotes the induced normalised Lebesgue measure of $r_{\xi}^{\pi} \mathbb{S}^{1}$ in $\pi^{\perp}$, which can be, of course, identified with $\mathrm{d} \sigma_{r_{\xi}^{\pi}}^{2}$. Then, by Plancherel's theorem in $\pi$ (with the normalisation for the Fourier transform taken in $\S 2$ ),

$$
\begin{aligned}
T_{n-2, n}\left(\widehat{g_{1} \mathrm{~d} \sigma^{n}} \overline{\overline{g_{2} \mathrm{~d} \sigma^{n}}}\right)(\pi, y) & =\int_{\pi} \widehat{g_{1} \mathrm{~d} \sigma^{n}}(x+y) \overline{\widehat{g_{2} \mathrm{~d} \sigma^{n}}}(x+y) \mathrm{d} \lambda_{\pi}(x) \\
& =(2 \pi)^{n-2} \int_{\left|\xi^{\pi}\right| \leqslant 1} \mathcal{F}^{\perp}\left(g_{1, \xi} \mathrm{d} \sigma_{r_{\xi}^{\pi}}^{\perp}\right)(y) \overline{\mathcal{F}^{\perp}\left(g_{2, \xi \pi} \mathrm{d} \sigma_{r_{\xi}^{\pi}}^{\perp}\right.}(y) \mathrm{d} \lambda_{\pi}\left(\xi^{\pi}\right) .
\end{aligned}
$$

A further application of Plancherel's theorem in $\pi^{\perp}$ yields

$$
\begin{aligned}
& \int_{\pi^{\perp}} \mid\left(-\Delta_{y}\right)^{1 / 4}\left.T_{n-2, n}\left(\widehat{\overline{g_{1} \mathrm{~d} \sigma^{n}}} \overline{\overline{g_{2} \mathrm{~d} \sigma^{n}}}\right)(\pi, y)\right|^{2} \mathrm{~d} \lambda_{\pi^{\perp}}(y) \\
&=(2 \pi)^{2(n-1)} \int_{\left|\xi^{\pi}\right| \leqslant 1} \int_{\left|\zeta^{\pi}\right| \leqslant 1} A_{\mathbb{S}^{n-1}, \pi}\left(\xi^{\pi}, \zeta^{\pi}\right) \mathrm{d} \lambda_{\pi}\left(\xi^{\pi}\right) \mathrm{d} \lambda_{\pi}\left(\zeta^{\pi}\right) .
\end{aligned}
$$

where

$$
A_{\mathbb{S}^{n-1}, \pi}\left(\xi^{\pi}, \zeta^{\pi}\right):=\int_{\pi^{\perp}}|v|\left(\tilde{g}_{1, \xi^{\pi}} \mathrm{d} \sigma_{r_{\xi}^{\pi}}^{\perp} *^{\perp} \bar{g}_{2, \xi^{\pi}} \mathrm{d} \sigma_{r_{\xi}^{\pi}}^{\perp}\right)(v)\left(\overline{\tilde{g}_{1, \zeta^{\pi}} \mathrm{d} \sigma_{r_{\zeta}^{\pi}}^{\perp} *^{\perp} \bar{g}_{2, \zeta^{\pi}} \mathrm{d} \sigma_{r_{\zeta}^{\pi}}^{\perp}}\right)(v) \mathrm{d} \lambda_{\pi^{\perp}}(v)
$$

and $\tilde{g}(\cdot)=g(-\cdot)$. For fixed $\xi^{\pi}, \zeta^{\pi}$ with $\left|\xi^{\pi}\right| \leqslant 1$ and $\left|\zeta^{\pi}\right| \leqslant 1, A_{\mathbb{S}^{n-1}, \pi}\left(\xi^{\pi}, \zeta^{\pi}\right)$ equals to

$$
\int_{\left(r_{\xi}^{\pi} \mathbb{S}^{1}\right)^{2} \times\left(r_{\zeta}^{\pi} \mathbb{S}^{1}\right)^{2}}\left|\xi^{\perp}\right|^{\perp / 2}\left|\zeta^{\perp}-\mu^{\perp}\right|^{1 / 2} g_{1, \xi^{\pi}}\left(\xi^{\perp}\right) \bar{g}_{2, \xi^{\pi}}\left(\eta^{\perp}\right) g_{2, \zeta^{\pi}}\left(\zeta^{\perp}\right) \bar{g}_{1, \zeta^{\pi}}\left(\mu^{\perp}\right) \mathrm{d} \Sigma_{\xi^{\pi}, \zeta^{\pi}}^{\perp}\left(\xi^{\perp}, \eta^{\perp}, \zeta^{\perp}, \mu^{\perp}\right)
$$

where

$$
\mathrm{d} \Sigma_{\xi^{\pi}, \zeta^{\pi}}^{\perp}\left(\xi^{\perp}, \eta^{\perp}, \zeta^{\perp}, \mu^{\perp}\right):=\delta\left(\xi^{\perp}+\zeta^{\perp}-\eta^{\perp}-\mu^{\perp}\right) \mathrm{d} \sigma_{r_{\xi}^{\pi}}^{\perp}\left(\xi^{\perp}\right) \mathrm{d} \sigma_{r_{\xi}^{\pi}}^{\perp}\left(\eta^{\perp}\right) \mathrm{d} \sigma_{r_{\zeta}^{\pi}}^{\perp}\left(\zeta^{\perp}\right) \mathrm{d} \sigma_{r_{\zeta}^{\pi}}^{\perp}\left(\mu^{\perp}\right) .
$$

Observe that one may rewrite the above integral as

$$
A_{\mathbb{S}^{n-1}, \pi}\left(\xi^{\pi}, \zeta^{\pi}\right)=\int_{r_{\xi}^{\pi} \mathbb{S}^{1} \times r_{\zeta}^{\pi} \mathbb{S}^{1}} g_{1, \xi^{\pi}}\left(\xi^{\perp}\right) g_{2, \zeta^{\pi}}\left(\zeta^{\perp}\right)\left(h_{2, \xi} \mathrm{d} \sigma_{r_{\xi}^{\pi}}^{\perp} *^{\perp} h_{1, \zeta} \mathrm{d} \sigma_{r_{\zeta}^{\pi}}^{\perp}\right)\left(\xi^{\perp}+\zeta^{\perp}\right) \mathrm{d} \sigma_{r_{\xi}^{\pi}}^{\perp}\left(\xi^{\perp}\right) \mathrm{d} \sigma_{r_{\zeta}^{\pi}}^{\perp}\left(\zeta^{\perp}\right)
$$

where $h_{2, \xi}\left(\eta^{\perp}\right):=\bar{g}_{2, \xi^{\pi}}\left(\eta^{\perp}\right)\left|\xi^{\perp}-\eta^{\perp}\right|^{1 / 2}$ and similarly for $h_{1, \zeta}$. As $\pi^{\perp} \cong \mathbb{R}^{2}$, assuming without loss of generality that $r_{\xi}^{\pi} \leqslant r_{\zeta}^{\pi}$, one can appeal to Lemma 3.1 to evaluate

$$
\left(h_{2, \xi} \mathrm{d} \sigma_{r_{\xi}^{\pi}}^{\perp} *^{\perp} h_{1, \zeta} \mathrm{d} \sigma_{r_{\zeta}^{\pi}}^{\perp}\right)\left(\xi^{\perp}+\zeta^{\perp}\right)=\frac{2 h_{2, \xi}\left(\xi^{\perp}\right) h_{1, \zeta}\left(\zeta^{\perp}\right)+2 h_{2, \xi}\left(\tilde{\xi}^{\perp}\right) h_{1, \zeta}\left(\tilde{\zeta}^{\perp}\right)}{\sqrt{-\left(\left|\xi^{\perp}+\zeta^{\perp}\right|^{2}-\left(r_{\zeta}^{\pi}+r_{\xi}^{\pi}\right)^{2}\right)\left(\left|\xi^{\perp}+\zeta^{\perp}\right|^{2}-\left(r_{\zeta}^{\pi}-r_{\xi}^{\pi}\right)^{2}\right)}}
$$

after noting that if $x=\xi^{\perp}+\zeta^{\perp}$ then $\left(P_{1}^{+}(x), P_{1}^{-}(x), P_{2}^{+}(x), P_{2}^{-}(x)\right)=\left(\xi^{\perp}, \tilde{\xi}^{\perp}, \zeta^{\perp}, \tilde{\zeta}^{\perp}\right)$, where $\tilde{\xi}^{\perp}, \tilde{\zeta}^{\perp} \in \pi^{\perp}$ are the reflected points of $\xi^{\perp}$ and $\zeta^{\perp}$ with respect to $\xi^{\perp}+\zeta^{\perp}$. Note that the implicit support condition $r_{\zeta}^{\pi}-r_{\xi}^{\pi} \leqslant\left|\xi^{\perp}+\zeta^{\perp}\right| \leqslant r_{\zeta}^{\pi}+r_{\xi}^{\pi}$ in (4.2) always holds under the assumption $r_{\xi}^{\pi} \leqslant r_{\zeta}^{\pi}$. Observe that $h_{2, \xi}\left(\xi^{\perp}\right)=h_{1, \zeta}\left(\zeta^{\perp}\right)=0$, so manipulating the denominator one has

$$
\left(h_{\xi} \mathrm{d} \sigma_{r_{\xi}^{\pi}}^{\perp} *^{\perp} h_{\zeta} \mathrm{d} \sigma_{r_{\zeta}^{\pi}}^{\perp}\right)\left(\xi^{\perp}+\zeta^{\perp}\right)=\left(\frac{\left|\xi^{\perp}-\tilde{\xi}^{\perp}\right|\left|\zeta^{\perp}-\tilde{\zeta}^{\perp}\right|}{\left(r_{\xi}^{\pi} r_{\zeta}^{\pi}\right)^{2}-\left(\xi^{\perp} \cdot \zeta^{\perp}\right)^{2}}\right)^{1 / 2} \bar{g}_{2, \xi^{\pi}}\left(\tilde{\xi}^{\perp}\right) \bar{g}_{1, \zeta^{\pi}}\left(\tilde{\zeta}^{\perp}\right)
$$

for all $\xi^{\perp} \in r_{\xi}^{\pi} \mathbb{S}^{1}$ and $\zeta^{\perp} \in r_{\zeta}^{\pi} \mathbb{S}^{1}$. Next note that $\left|\xi^{\perp} \wedge \zeta^{\perp}\right|^{2}=\left(r_{\xi}^{\pi} r_{\zeta}^{\pi}\right)^{2}-\left(\xi^{\perp} \cdot \zeta^{\perp}\right)^{2}$, but also $\left|\xi^{\perp} \wedge \zeta^{\perp}\right|^{2}=\frac{1}{4}\left|\xi^{\perp}+\zeta^{\perp}\right|^{2}\left|\xi^{\perp}-\tilde{\xi}^{\perp}\right|\left|\zeta^{\perp}-\tilde{\zeta}^{\perp}\right|$, as the points satisfy the relation $\xi^{\perp}+\zeta^{\perp}=\tilde{\xi}^{\perp}+\tilde{\zeta}^{\perp}$. Thus,

$$
\left(\frac{\left|\xi^{\perp}-\tilde{\xi}^{\perp}\right|\left|\zeta^{\perp}-\tilde{\zeta}^{\perp}\right|}{\left(r_{\xi}^{\pi} r_{\zeta}^{\pi}\right)^{2}-\left(\xi^{\perp} \cdot \zeta^{\perp}\right)^{2}}\right)^{1 / 2}=\frac{2}{\left|\xi^{\perp}+\zeta^{\perp}\right|}
$$


and combining the above estimates one obtains

$$
\begin{aligned}
& \int_{\pi^{\perp}}\left|\left(-\Delta_{y}\right)^{1 / 4} T_{n-2, n}\left(\widehat{g_{1} \mathrm{~d} \sigma^{n}} \overline{\widehat{g_{2} \mathrm{~d} \sigma^{n}}}\right)(\pi, y)\right|^{2} \mathrm{~d} \lambda_{\pi^{\perp}}(y) \\
& =(2 \pi)^{2(n-1)} \int_{\left|\xi^{\pi}\right| \leqslant 1} \int_{\left|\zeta^{\pi}\right| \leqslant 1} \int_{r_{\xi}^{\pi} \mathbb{S}^{1} \times r_{\zeta}^{\pi} \mathbb{S}^{1}} K_{\pi, \mathbb{S}^{n-1}}(\xi, \zeta) g_{1, \xi^{\pi}}\left(\xi^{\perp}\right) g_{2, \zeta^{\pi}}\left(\zeta^{\perp}\right) \bar{g}_{2, \xi^{\pi}}\left(\tilde{\xi}^{\perp}\right) \bar{g}_{1, \zeta^{\pi}}\left(\tilde{\zeta}^{\perp}\right) \mathrm{d} \Sigma_{\pi}(\xi, \zeta) \\
& =(2 \pi)^{2(n-1)} \int_{\left(\mathbb{S}^{n-1}\right)^{2}} K_{\pi, \mathbb{S}^{n-1}}(\xi, \zeta) g_{1}(\xi) g_{2}(\zeta) \bar{g}_{2}\left(\xi^{\pi}+\tilde{\xi}^{\perp}\right) \bar{g}_{1}\left(\zeta^{\pi}+\tilde{\zeta}^{\perp}\right) \mathrm{d} \sigma^{n}(\xi) \mathrm{d} \sigma^{n}(\zeta),
\end{aligned}
$$

completing the proof of Theorem 1.2; above $\mathrm{d} \Sigma_{\pi}(\xi, \zeta):=\mathrm{d} \sigma_{r_{\xi}^{\pi}}^{\perp}\left(\xi^{\perp}\right) \mathrm{d} \sigma_{r_{\zeta}^{\pi}}^{\perp}\left(\zeta^{\perp}\right) \mathrm{d} \lambda_{\pi}\left(\xi^{\pi}\right) \mathrm{d} \lambda_{\pi}\left(\zeta^{\pi}\right)$.

4.2. Proof of Theorem 1.6. Given $\omega \in \mathbb{S}_{+}^{d-1}$ and $\pi=\langle\omega\rangle^{\perp} \in \mathcal{G}_{d-1, d}$ write, for each $\xi \in \mathbb{R}^{d}$, $\xi=\xi^{\pi}+\xi^{\omega} \omega$, where $\xi^{\omega}=\xi \cdot \omega$ and let $m_{\xi}^{\pi}:=\sqrt{m^{2}+\left|\xi^{\pi}\right|^{2}}$. Given $s \in \mathbb{R}$ and $x \in \pi$,

$$
\begin{aligned}
\widehat{g_{j} \mathrm{~d} \sigma_{\mathbb{H}_{m}^{d}}}(x+s \omega, t) & =\int_{\mathbb{R}^{d}} e^{i(x+s \omega) \cdot \xi+i t \sqrt{m^{2}+|\xi|^{2}}} f_{j}(\xi) \frac{\mathrm{d} \xi}{\sqrt{m^{2}+|\xi|^{2}}} \\
& =\int_{\pi} e^{i x \cdot \xi^{\pi}} \int_{\mathbb{R}} e^{i s \xi^{\omega}+i t \sqrt{m^{2}+\left|\xi^{\pi}\right|^{2}+\left|\xi^{\omega}\right|^{2}}} f_{j}\left(\xi^{\pi}+\xi^{\omega} \omega\right) \frac{\mathrm{d} \xi^{\omega}}{\sqrt{m^{2}+\left|\xi^{\pi}\right|^{2}+\left|\xi^{\omega}\right|^{2}}} \mathrm{~d} \lambda_{\pi}\left(\xi^{\pi}\right) \\
& =\int_{\pi} e^{i x \cdot \xi^{\pi}} \mathcal{F}^{2}\left(g_{j, \xi^{\pi}} \mathrm{d} \sigma_{\mathbb{H}_{m_{\xi}^{1}}^{1}}\right)(s, t) \mathrm{d} \lambda_{\pi}\left(\xi^{\pi}\right),
\end{aligned}
$$

where $f_{j, \xi^{\pi}}(\nu):=f_{j}\left(\xi^{\pi}+\nu \omega\right)$ for all $\nu \in \mathbb{R}$ and $g_{j, \xi^{\pi}}$ denotes the lift of $f_{j, \xi^{\pi}}$ to $\mathbb{H}_{m_{\xi}^{\pi}}^{1}$, and $\mathcal{F}^{2}$ denotes the 2-dimensional Fourier transform. Reasoning as in the proof of Theorem 1.2,

$\left.\int_{\mathbb{R}} \int_{\mathbb{R}} \mid \partial_{s}^{1 / 2} \mathcal{R}\left(g_{1} \widehat{\mathrm{d} \sigma_{\mathbb{H}_{m}^{d}}^{d}}\right)(\cdot, t) \widehat{\widehat{g_{2} \mathrm{~d} \sigma_{\mathbb{H}_{m}^{d}}^{d}}}(\cdot, t)\right)\left.(\omega, s)\right|^{2} \mathrm{~d} s \mathrm{~d} t=(2 \pi)^{2 d} \int_{\pi} \int_{\pi} A_{\mathbb{H}_{m}^{d}, \pi}\left(\xi^{\pi}, \zeta^{\pi}\right) \mathrm{d} \lambda_{\pi}\left(\xi^{\pi}\right) \mathrm{d} \lambda_{\pi}\left(\zeta^{\pi}\right)$, where

$$
A_{\mathbb{H}_{m}^{d}, \pi}\left(\xi^{\pi}, \zeta^{\pi}\right):=\int_{\mathbb{R}} \int_{\mathbb{R}}|v|\left(\tilde{g}_{1, \xi^{\pi}} \mathrm{d} \sigma_{\mathbb{H}_{m_{\xi}^{\pi}}^{1}} *^{2} \bar{g}_{2, \xi^{\pi}} \mathrm{d} \sigma_{\mathbb{H}_{m_{\xi}^{\pi}}^{1}}\right)(v, \tau) \overline{\left(\tilde{g}_{1, \zeta^{\pi}} \mathrm{d} \sigma_{\mathbb{H}_{m_{\zeta}^{\pi}}^{1}} *^{2} \bar{g}_{2, \zeta^{\pi}} \mathrm{d} \sigma_{\mathbb{H}_{m_{\zeta}^{1}}^{1}}\right)}(v, \tau) \mathrm{d} v \mathrm{~d} \tau
$$

Note that

$$
A_{\mathbb{H}_{m}^{d}, \pi}\left(\xi^{\pi}, \zeta^{\pi}\right)=\int_{\mathbb{R}} \int_{\mathbb{R}} f_{1, \xi^{\pi}}\left(\xi^{\omega}\right) f_{2, \zeta^{\pi}}\left(\zeta^{\omega}\right)\left(H_{2, \xi} \mathrm{d} \sigma_{\mathbb{H}_{m_{\xi}^{1}}^{1}} * H_{1, \zeta} \mathrm{d}{\sigma_{\mathbb{H}_{m_{\zeta}^{\pi}}^{1}}}_{l}\right)\left(P_{\xi, \zeta, \omega}\right) \frac{\mathrm{d} \xi^{\omega}}{\phi_{m_{\xi}^{\pi}}\left(\xi^{\omega}\right)} \frac{\mathrm{d} \zeta^{\omega}}{\phi_{m_{\zeta}^{\pi}}\left(\zeta^{\omega}\right)},
$$

where $H_{2, \xi}$ is the lift of $h_{2, \xi}(\eta):=\bar{f}_{2, \xi^{\pi}}(\eta)\left|\xi^{\omega}-\eta\right|^{1 / 2}$ to $\mathbb{H}_{m_{\xi}^{\pi}}^{1}\left(\right.$ similarly for $\left.H_{1, \zeta}\right)$ and $P_{\xi, \zeta, \omega}$ denotes the point

$$
P_{\xi, \zeta, \omega}:=\left(\xi^{\omega}+\zeta^{\omega}, \phi_{m_{\xi}^{\pi}}\left(\xi^{\omega}\right)+\phi_{m_{\zeta}^{\pi}}\left(\zeta^{\omega}\right)\right) .
$$

Denoting by $r_{P}$ the hyperbolic radius of $P_{\xi, \zeta, \omega}$, that is, $r_{P}^{2}=\left(\phi_{m_{\xi}^{\pi}}\left(\xi^{\omega}\right)+\phi_{m_{\zeta}^{\pi}}\left(\zeta^{\omega}\right)\right)^{2}-\left(\xi^{\omega}+\zeta^{\omega}\right)^{2}$, Lemma 3.2 yields

$$
\left(H_{2, \xi} \mathrm{d} \sigma_{\mathbb{H}_{m_{\xi}^{\pi}}^{1}} * H_{1, \zeta} \mathrm{d}{\sigma_{\mathbb{H}_{m_{\zeta}^{\pi}}^{1}}}\right)\left(P_{\xi, \zeta, \omega}\right)=\frac{2 h_{2, \xi}\left(\xi^{\omega}\right) h_{1, \zeta}\left(\zeta^{\omega}\right)+2 h_{2, \xi}\left(\tilde{\xi}^{\omega}\right) h_{1, \zeta}\left(\tilde{\zeta}^{\omega}\right)}{\sqrt{r_{P}^{4}-2 r_{P}^{2}\left(\left(m_{\xi}^{\pi}\right)^{2}+\left(m_{\zeta}^{\pi}\right)^{2}\right)+\left(\left(m_{\xi}^{\pi}\right)^{2}-\left(m_{\zeta}^{\pi}\right)^{2}\right)^{2}}}
$$

where $\left(\tilde{\xi}^{\omega}, \phi_{m_{\xi}^{\pi}}\left(\tilde{\xi}^{\omega}\right)\right)=Q_{1}^{-}\left(P_{\xi, \zeta, \omega}\right) \in \mathbb{H}_{m_{\xi}^{\pi}}^{1}$ and $\left(\tilde{\zeta}^{\omega}, \phi_{m_{\zeta}^{\pi}}\left(\tilde{\zeta}^{\omega}\right)\right)=Q_{2}^{+}\left(P_{\xi, \zeta, \omega}\right) \in \mathbb{H}_{m_{\zeta}^{\pi}}^{1}$. After an algebraic manipulation and noting that $h_{1, \zeta}\left(\zeta^{\omega}\right)=h_{2, \xi}\left(\xi^{\omega}\right)=0,(4.4)$ becomes

$$
\left(H_{2, \xi} \mathrm{d} \sigma_{\mathbb{H}_{m \frac{\pi}{\pi}}^{1}} * H_{1, \zeta} \mathrm{d} \sigma_{\mathbb{H}_{m \frac{\pi}{\zeta}}^{1}}\right)\left(P_{\xi, \zeta, \omega}\right)=\frac{\left|\xi^{\omega}-\tilde{\xi}^{\omega}\right|^{1 / 2} \bar{f}_{2, \xi^{\pi}}\left(\tilde{\xi}^{\omega}\right)\left|\zeta^{\omega}-\tilde{\zeta}^{\omega}\right|^{1 / 2} \bar{f}_{1, \zeta^{\pi}}\left(\tilde{\zeta}^{\omega}\right)}{\left|\xi^{\omega} \phi_{m_{\zeta}^{\pi}}\left(\zeta^{\omega}\right)-\zeta^{\omega} \phi_{m_{\xi}^{\pi}}\left(\xi^{\omega}\right)\right|} .
$$

Putting all the estimates together as in the proof of Theorem 1.2 concludes the proof.

Remark 4.1. As the points in the pairs $\left(Q_{1}^{+}(0, z), Q_{1}^{-}(0, z)\right)$ and $\left(Q_{2}^{+}(0, z), Q_{2}^{-}(0, z)\right)$ are symmetric with respect to the vertical axis, it is a simple exercise to obtain an expression for $\tilde{\xi}^{\omega}$ and 
$\tilde{\zeta}^{\omega}$ via Lorentz transformations. Indeed, let $\gamma_{P}$ denote the hyperbolic angle of $P_{\xi, \zeta, \omega}$ and let $L_{\gamma_{P}}$ denote, as in (2.3), the Lorentz transformation such that $L_{\gamma_{P}}\left(P_{\xi, \zeta, \omega}\right)=\left(0, r_{P}\right)$. Then

$$
\begin{aligned}
& Q_{1}^{+}\left(0, r_{P}\right)=L_{\gamma_{P}}\left(\xi^{\omega}, \phi_{m_{\xi}^{\pi}}\left(\xi^{\omega}\right)\right)=\left(m_{\xi}^{\pi} \sinh \left(\gamma_{\xi}-\gamma_{P}\right), m_{\xi}^{\pi} \cosh \left(\gamma_{\xi}-\gamma_{P}\right)\right) \\
& Q_{2}^{-}\left(0, r_{P}\right)=L_{\gamma_{P}}\left(\zeta^{\omega}, \phi_{m_{\zeta}^{\pi}}\left(\zeta^{\omega}\right)\right)=\left(m_{\zeta}^{\pi} \sinh \left(\gamma_{\zeta}-\gamma_{P}\right), m_{\zeta}^{\pi} \cosh \left(\gamma_{\zeta}-\gamma_{P}\right)\right) .
\end{aligned}
$$

Clearly,

$$
\begin{aligned}
& Q_{1}^{-}\left(0, r_{P}\right)=\left(-m_{\xi}^{\pi} \sinh \left(\gamma_{\xi}-\gamma_{P}\right), m_{\xi}^{\pi} \cosh \left(\gamma_{\xi}-\gamma_{P}\right)\right) \\
& Q_{2}^{+}\left(0, r_{P}\right)=\left(-m_{\zeta}^{\pi} \sinh \left(\gamma_{\zeta}-\gamma_{P}\right), m_{\zeta}^{\pi} \cosh \left(\gamma_{\zeta}-\gamma_{P}\right)\right)
\end{aligned}
$$

and

$$
\begin{aligned}
& Q_{1}^{-}\left(P_{\xi, \zeta, \omega}\right)=L_{-\gamma_{P}}\left(Q_{1}^{-}\left(0, r_{P}\right)\right)=\left(m_{\xi}^{\pi} \sinh \left(2 \gamma_{P}-\gamma_{\xi}\right), m_{\xi}^{\pi} \cosh \left(2 \gamma_{P}-\gamma_{\xi}\right)\right) \\
& Q_{2}^{+}\left(P_{\xi, \zeta, \omega}\right)=L_{-\gamma_{P}}\left(Q_{2}^{+}\left(0, r_{P}\right)\right)=\left(m_{\zeta}^{\pi} \sinh \left(2 \gamma_{P}-\gamma_{\zeta}\right), m_{\zeta}^{\pi} \cosh \left(2 \gamma_{P}-\gamma_{\zeta}\right)\right),
\end{aligned}
$$

so $\tilde{\xi}^{\omega}=m_{\xi}^{\pi} \sinh \left(2 \gamma_{P}-\gamma_{\xi}\right)$ and $\tilde{\zeta}^{\omega}=m_{\zeta}^{\pi} \sinh \left(2 \gamma_{P}-\gamma_{\zeta}\right)$. In particular, this allows one to rewrite the kernel as

$$
K_{\omega, \mathbb{H}_{m}^{d}}(\xi, \zeta)=\frac{\left(m_{\xi}^{\omega} m_{\zeta}^{\omega}\left|\sinh \left(\gamma_{\xi}-\gamma_{P}\right)\right|\left|\cosh \left(\gamma_{\xi}+\gamma_{P}\right)\right|\left|\sinh \left(\gamma_{\zeta}-\gamma_{P}\right) \| \cosh \left(\gamma_{\zeta}+\gamma_{P}\right)\right|\right)^{1 / 2}}{m_{\xi}^{\omega} m_{\zeta}^{\omega}\left|\sinh \left(\gamma_{\xi}-\gamma_{\zeta}\right)\right|}
$$

Remark 4.2. Note that

$$
\left|\xi^{\omega}-\tilde{\xi}^{\omega}\right|=\mid\left(L_{\gamma_{P}}^{-1}\left(L_{\gamma_{P}}\left(\xi^{\omega}, \phi_{m_{\xi}^{\pi}}\left(\xi^{\omega}\right)\right)-L_{\gamma_{P}}\left(\left(\tilde{\xi}^{\omega}, \phi_{m_{\xi}^{\pi}}\left(\tilde{\xi}^{\omega}\right)\right)\right)\right)_{1}|=|\left(L_{\gamma_{P}}^{-1}(2 a, 0)\right)_{1}|=2| a \mid \cosh \left(\gamma_{P}\right),\right.
$$

where $a:=m_{\xi}^{\pi} \sinh \left(\gamma_{\xi}-\gamma_{P}\right)$. As $\left|\xi^{\omega}-\tilde{\xi}^{\omega}\right|=\left|\zeta^{\omega}-\tilde{\zeta}^{\omega}\right|$ and the denominator in (4.4) is easily seen to be equal to $|a| r_{P}$ (see the proof of Lemma 3.2), the kernel $K_{\omega, \mathbb{H}_{m}^{d}}$ may then be expressed as

$$
K_{\omega, \mathbb{H}_{m}^{d}}=\frac{2\left(\phi_{m_{\xi}^{\pi}}\left(\xi^{\omega}\right)+\phi_{m_{\zeta}^{\pi}}\left(\zeta^{\omega}\right)\right)}{\left(\phi_{m_{\xi}^{\pi}}\left(\xi^{\omega}\right)+\phi_{m_{\zeta}^{\pi}}\left(\zeta^{\omega}\right)\right)^{2}-\left(\xi^{\omega}+\zeta^{\omega}\right)^{2}}
$$

after noting that $\cosh \left(\gamma_{P}\right)=\left(\phi_{m_{\xi}^{\pi}}\left(\xi^{\omega}\right)+\phi_{m_{\zeta}^{\pi}}\left(\zeta^{\omega}\right)\right) / r_{P}$.

\section{Corollaries}

5.1. Proof of Corollary 1.3. By (1.11) it is clear that the expression

$$
g_{1}(\xi) \bar{g}_{2}\left(\xi^{\pi}+\tilde{\xi}^{\perp}\right) g_{2}(\zeta) \bar{g}_{1}\left(\zeta^{\pi}+\tilde{\zeta}^{\perp}\right)
$$

on its right-hand side is real and positive. The identity (1.12) then yields that (5.1) equals to

$$
\frac{1}{2}\left(\left|g_{1}(\xi) g_{2}(\zeta)\right|^{2}+\left|g_{2}\left(\xi^{\pi}+\tilde{\xi}^{\perp}\right) g_{1}\left(\zeta^{\pi}+\tilde{\zeta}^{\perp}\right)\right|^{2}-\left|g_{1}(\xi) g_{2}(\zeta)-g_{2}\left(\xi^{\pi}+\tilde{\xi}^{\perp}\right) g_{1}\left(\zeta^{\pi}+\tilde{\zeta}^{\perp}\right)\right|^{2}\right) .
$$

The negative term above immediately gives raise to the expression $I_{\pi, \mathbb{S}^{n-1}}\left(g_{1}, g_{2}\right)$, whilst the positive terms amount to the same expression over the integral sign, finishing the proof.

Remark 5.1. Observe that the resulting sharp inequality

$$
\int_{\pi^{\perp}}\left|\left(-\Delta_{y}\right)^{1 / 4} T_{n-2, n}\left(\widehat{\overline{g_{1} \mathrm{~d} \sigma^{n}}} \overline{\overline{g_{2} \mathrm{~d} \sigma^{n}}}\right)(\pi, y)\right|^{2} \mathrm{~d} y \leqslant \mathbf{C}_{\mathbb{S}^{n-1}} \int_{\left.\mathbb{S}^{n-1}\right)^{2}} K_{\pi, \mathbb{S}^{n-1}}(\xi, \zeta)\left|g_{1}(\xi)\right|^{2}\left|g_{2}(\zeta)\right|^{2} \mathrm{~d} \sigma^{n}(\xi) \mathrm{d} \sigma^{n}(\zeta)
$$

obtained from dropping the negative term in (1.13) may be deduced more directly via a simple application of the Cauchy-Schwarz inequality. Note that (4.1) is a positive quantity, so in particular equals to its modulus. By the triangle inequality, the left-hand side of (1.11) is controlled by

$$
\begin{array}{r}
\int_{\left|\xi^{\pi}\right| \leqslant 1} \int_{\left|\zeta^{\pi}\right| \leqslant 1} \int_{\left(r_{\xi}^{\pi} \mathbb{S}^{1}\right)^{2} \times\left(r_{\zeta}^{\pi} \mathbb{S}^{1}\right)^{2}}\left|\xi^{\perp} \eta^{\perp}\right|^{1 / 2}\left|\zeta^{\perp}-\mu^{\perp}\right|^{1 / 2}\left|g_{1, \xi^{\pi}}\left(\xi^{\perp}\right)\right|\left|g_{2, \xi^{\pi}}\left(\eta^{\perp}\right)\right|\left|g_{2, \zeta^{\pi}}\left(\zeta^{\perp}\right)\right|\left|g_{1, \zeta^{\pi}}\left(\mu^{\perp}\right)\right| \\
\mathrm{d} \Sigma_{\xi^{\pi}, \zeta^{\pi}}^{\perp}\left(\xi^{\perp}, \eta^{\perp}, \zeta^{\perp}, \mu^{\perp}\right) \mathrm{d} \lambda_{\pi}\left(\xi^{\pi}\right) \mathrm{d} \lambda_{\pi}\left(\zeta^{\pi}\right) .
\end{array}
$$


Applying the Cauchy-Schwarz inequality with respect to the measure $\mathrm{d} \Sigma_{\xi^{\pi}, \zeta^{\pi}}^{\perp} \mathrm{d} \lambda_{\pi}\left(\xi^{\pi}\right) \mathrm{d} \lambda_{\pi}\left(\zeta^{\pi}\right)$, the above is further controlled by

$$
\int_{\left|\xi^{\pi}\right| \leqslant 1} \int_{\left|\zeta^{\pi}\right| \leqslant 1} \int_{r_{\xi}^{\pi} \mathbb{S}^{1} \times r_{\zeta}^{\pi} \mathbb{S}^{1}}\left|g_{1, \xi^{\pi}}\left(\xi^{\perp}\right)\right|^{2}\left|g_{2, \zeta^{\pi}}\left(\zeta^{\perp}\right)\right|^{2}\left(h_{\xi} \mathrm{d} \sigma_{r_{\xi}^{\pi}}^{\perp} *^{\perp} h_{\zeta} \mathrm{d} \sigma_{r_{\zeta}^{\pi}}^{\perp}\right)\left(\xi^{\perp}+\zeta^{\perp}\right) \mathrm{d} \sigma_{r_{\xi}^{\pi}}^{\perp}\left(\xi^{\perp}\right) \mathrm{d} \Sigma_{\pi}(\xi, \zeta)
$$

where $h_{\xi}\left(\eta^{\perp}\right):=\left|\xi^{\perp}-\eta^{\perp}\right|^{1 / 2}$ and similarly for $h_{\zeta} ;$ above $\mathrm{d} \Sigma_{\pi}(\xi, \zeta):=\mathrm{d} \sigma_{r_{\zeta}^{\pi}}^{\perp}\left(\zeta^{\perp}\right) \mathrm{d} \lambda_{\pi}\left(\xi^{\pi}\right) \mathrm{d} \lambda_{\pi}\left(\zeta^{\pi}\right)$. Evaluation of the innermost convolution as in (4.3) yields then the desired inequality (5.2).

5.2. Proof of Corollary 1.4. Given $\pi \in \mathcal{G}_{n-2, n}$, Plancherel's theorem and the relation (2.1) yields

$$
\int_{\pi^{\perp}}\left|\left(-\Delta_{y}\right)^{1 / 4} T_{n-2, n} h(\pi, y)\right|^{2} \mathrm{~d} \lambda_{\pi^{\perp}}(y)=(2 \pi)^{-2} \int_{\pi^{\perp}}\left|\xi^{\perp}\right|\left|\widehat{h}\left(\xi^{\perp}\right)\right|^{2} \mathrm{~d} \lambda_{\pi^{\perp}}\left(\xi^{\perp}\right) .
$$

Averaging over all $\pi \in \mathcal{G}_{n-2, n}$, and using (2.2) and polar coordinates

$$
\begin{aligned}
\int_{\mathcal{G}_{n-2, n}} \int_{\pi^{\perp}}\left|\xi^{\perp}\right|^{3-n}\left|\widehat{h}\left(\xi^{\perp}\right)\right|^{2}\left|\xi^{\perp}\right|^{n-2} \mathrm{~d} \lambda_{\pi^{\perp}}\left(\xi^{\perp}\right) \mathrm{d} \mu_{\mathcal{G}}(\pi) \\
\quad=\int_{\mathcal{G}_{n-2, n}} \int_{0}^{\infty} \int_{\mathbb{S}^{n-1} \cap \pi^{\perp}} r^{3-n}|\widehat{h}(r \omega)|^{2} r^{n-2} r \mathrm{~d} r \mathrm{~d} \sigma^{n, \perp}(\omega) \mathrm{d} \mu_{\mathcal{G}}(\pi) \\
=\left|\mathcal{G}_{1, n-1}\right| \int_{0}^{\infty} \int_{\mathbb{S}^{n-1}} r^{3-n}|\widehat{h}(r \omega)|^{2} r^{n-1} \mathrm{~d} r \mathrm{~d} \sigma^{n}(\omega) \\
=\left.\left.\left|\mathcal{G}_{1, n-1}\right|(2 \pi)^{n} \int_{\mathbb{R}^{n}}|| \nabla\right|^{\frac{3-n}{2}} h(x)\right|^{2} \mathrm{~d} x,
\end{aligned}
$$

which completes the proof on taking $h=\widehat{g_{1} \mathrm{~d} \sigma^{n}} \widehat{\overline{g_{2} \mathrm{~d} \sigma^{n}}}$.

5.3. Proof of Corollary 1.5. Recall $\xi=\xi^{\pi}+\xi^{\perp}$. For $n=3, \pi=\langle\omega\rangle$, where $\omega \in \mathcal{G}_{1,3} \simeq \mathbb{S}_{+}^{2}$. Then $\xi^{\pi}=(\xi \cdot \omega) \omega$ and $\xi^{\perp}=\xi-(\xi \cdot \omega) \omega$, so

$$
\left|\xi^{\perp}+\zeta^{\perp}\right|^{2}=|\xi+\zeta|^{2}+|(\xi+\zeta) \cdot \omega|^{2}-2((\xi+\zeta) \cdot \omega)^{2}=|\xi+\zeta|^{2}\left(1-\left(\frac{(\xi+\zeta)}{|\xi+\zeta|} \cdot \omega\right)^{2}\right) .
$$

Noting that $\left|\mathcal{G}_{1,2}\right|=\pi$,

$$
K_{\mathbb{S}^{n-1}}(\xi, \zeta)=\frac{2}{\left|\mathcal{G}_{1,2}\right|} \int_{\mathbb{S}_{+}^{2}} \frac{\mathrm{d} \sigma_{+}^{3}(\omega)}{\left|\xi^{\perp}+\zeta^{\perp}\right|}=\frac{2 \pi}{\pi|\xi+\zeta|} \int_{-1}^{1} \frac{\mathrm{d} u}{\sqrt{1-u^{2}}}=\frac{2 \pi}{|\xi+\zeta|} .
$$

Thus

$$
\left\|\widehat{g \mathrm{~d} \sigma^{3}}\right\|_{L^{4}\left(\mathbb{R}^{3}\right)}^{4} \leqslant(2 \pi)^{4} \int_{\mathbb{S}^{2}} \int_{\mathbb{S}^{2}} \frac{1}{|\xi+\zeta|}|g(\xi)|^{2}|g(\zeta)|^{2} \mathrm{~d} \sigma^{3}(\xi) \mathrm{d} \sigma^{3}(\zeta)
$$

and the desired sharp Stein-Tomas inequality for the sphere follows from the following fact due to Foschi [19]:

$$
\int_{\mathbb{S}^{2}} \int_{\mathbb{S}^{2}} \frac{1}{|\xi+\zeta|}|g(\xi)|^{2}|g(\zeta)|^{2} \mathrm{~d} \sigma^{3}(\xi) \mathrm{d} \sigma^{3}(\zeta) \leqslant\|g\|_{L^{2}\left(\mathbb{S}^{2}\right)}^{4},
$$

which holds for $g$ antipodally symmetric. The reduction to the antipodally symmetric case may be done as in [19], using the Cauchy-Schwarz inequality for real numbers

$$
a c+b d \leqslant \sqrt{a^{2}+b^{2}} \sqrt{c^{2}+d^{2}} .
$$

Indeed, note that in the proof of (5.2) via the Cauchy-Schwarz inequality given in Section 5.1, one may replace $\left|g_{\xi^{\pi}}\left(\xi^{\perp}\right)\right|\left|g_{\xi^{\pi}}\left(\eta^{\perp}\right)\right|$ in the innermost integral in (5.3) by

$$
\frac{\left|g_{\xi^{\pi}}\left(\xi^{\perp}\right)\right|\left|g_{\xi^{\pi}}\left(\eta^{\perp}\right)\right|+\left|g_{\xi^{\pi}}\left(-\xi^{\perp}\right)\right|\left|g_{\xi^{\pi}}\left(-\eta^{\perp}\right)\right|}{2},
$$


and using (5.5) this is bounded by $\left|g_{\xi^{\pi}}^{\#}\left(\xi^{\perp}\right)\right|\left|g_{\xi^{\pi}}^{\#}\left(\eta^{\perp}\right)\right|$, where for any function $h$, the function $h^{\#}$ denotes $h^{\#}(\xi):=\sqrt{(h(\xi)+h(-\xi)) / 2}$, which is antipodally symmetric. One can argue similarly to replace $\left|g_{\zeta^{\pi}}\left(\zeta^{\perp}\right)\right|\left|g_{\zeta^{\pi}}\left(\mu^{\perp}\right)\right|$ by $\left|g_{\zeta^{\pi}}^{\#}\left(\zeta^{\perp}\right)\right|\left|g_{\zeta^{\pi}}^{\#}\left(\mu^{\perp}\right)\right|$. Thus, the right-hand side in (5.2) is replaced by $(5.6)$

$$
\mathbf{C}_{\mathbb{S}^{n-1}} \int_{\left|\xi^{\pi}\right| \leqslant 1} \int_{\left|\zeta^{\pi}\right| \leqslant 1} \int_{r_{\xi}^{\pi} \mathbb{S}^{1} \times r_{\zeta}^{\pi} \mathbb{S}^{1}} \frac{2}{\left|\xi^{\perp}+\zeta^{\perp}\right|}\left|g_{\xi^{\pi}}^{\#}\left(\xi^{\perp}\right)\right|^{2}\left|g_{\zeta^{\pi}}^{\#}\left(\zeta^{\perp}\right)\right|^{2} \mathrm{~d} \sigma_{r_{\xi^{\pi}}}^{1}\left(\xi^{\perp}\right) \mathrm{d} \sigma_{r_{\zeta^{\pi}}}^{1}\left(\zeta^{\perp}\right) \mathrm{d} \lambda_{\pi}\left(\xi^{\pi}\right) \mathrm{d} \lambda_{\pi}\left(\zeta^{\pi}\right) .
$$

One desires, however, to have $g^{\#}$ rather than $g_{\xi^{\pi}}^{\#}$ and $g_{\zeta^{\pi}}^{\#}$. By a change of variables, the integrand $4\left|g_{\xi^{\pi}}^{\#}\left(\xi^{\perp}\right)\right|^{2}\left|g_{\zeta^{\pi}}^{\#}\left(\zeta^{\perp}\right)\right|^{2}$ may be further replaced by

$$
\left(\left|g_{\xi^{\pi}}^{\#}\left(\xi^{\perp}\right)\right|^{2}+\left|g_{-\xi^{\pi}}^{\#}\left(\xi^{\perp}\right)\right|^{2}\right)\left(\left|g_{\zeta^{\pi}}^{\#}\left(\zeta^{\perp}\right)\right|^{2}+\left|g_{-\zeta^{\pi}}^{\#}\left(\zeta^{\perp}\right)\right|^{2}\right)
$$

which equals

$$
\left|g^{\#}(\xi)\right|^{2}\left|g^{\#}(\zeta)\right|^{2}+\left|g^{\#}(\xi)\right|^{2}\left|g^{\#}\left(\zeta^{\perp}-\zeta^{\pi}\right)\right|^{2}+\left|g^{\#}\left(\xi^{\perp}-\xi^{\pi}\right)\right|^{2}\left|g^{\#}(\zeta)\right|^{2}+\left|g^{\#}\left(\xi^{\perp}-\xi^{\pi}\right)\right|^{2}\left|g^{\#}\left(\zeta^{\perp}-\zeta^{\pi}\right)\right|^{2}
$$

A further change of variables in each of the terms allows one to see that (5.6) equals

$$
\mathbf{C}_{\mathbb{S}^{n-1}} \int_{\left(\mathbb{S}^{n-1}\right)^{2}} K_{\pi, \mathbb{S}^{n-1}}(\xi, \zeta)\left|g^{\#}(\xi)\right|^{2}\left|g^{\#}(\zeta)\right|^{2} \mathrm{~d} \sigma^{n}(\xi) \mathrm{d} \sigma^{n}(\zeta),
$$

as desired for the later application of Foschi's identity (5.4) on antipodally symmetric functions.

5.4. Proof of Corollary 1.7. This follows the same argument as that of Corollary 1.3.

5.5. Proof of Corollary 1.8. The proof follows from the same argument as in $\S 5.2$. Indeed, the elementary argument therein yields the relation

$$
\left\|(-\Delta)^{\ell / 2} f\right\|_{L^{2}\left(\mathbb{R}^{d}\right)}^{2}=(2 \pi)^{-(d-1)}\left\|\partial_{s}^{\frac{d-1}{2}+\ell} \mathcal{R} f\right\|_{L_{\omega, s}^{2}\left(\mathbb{S}_{+}^{d-1}, \mathbb{R}\right)}^{2},
$$

from which Corollary 1.8 follows from taking $\ell=(2-d) / 2$ after averaging over $\omega \in \mathbb{S}_{+}^{d-1}$; note that $\omega$ in the Radon transform $\mathcal{R}$ only runs over $\mathbb{S}_{+}^{d-1} \simeq \mathcal{G}_{d-1, d}$.

\section{The BILINEAR IDENTITy (1.10) FOR PARABOlOIDS REVISITED}

The purpose of this final section is to provide an alternative proof of Theorem 1.1 via Fourier analysis. The proof follows the same scheme as those of Theorems 1.2 and 1.6 with a little twist, which is available when taking one full derivative in the $s$-variable in the case of paraboloids.

To see this, let $\mathbb{P}_{a}^{d}:=\left\{\left(\xi,|\xi|^{2}+a\right): \xi \in \mathbb{R}^{d}\right\}$ denote the paraboloid in $(x, t) \in \mathbb{R}^{d} \times \mathbb{R}$ with tangent plane $t=a$ at its vertex; if $a=0$ we simply denote it by $\mathbb{P}^{d}$. Let $\mathrm{d} \sigma_{\mathbb{P}_{a}^{d}}$ denote the parametrised measure on $\mathbb{P}_{a}^{d}$, which satisfies $\widehat{g \mathrm{~d} \sigma_{\mathbb{P}_{a}^{d}}}(x, t)=E f(x, t)$ where $E$ is the extension operator associated to $\phi(\xi):=|\xi|^{2}+a$ and $g$ is the lift of the function $f: \mathbb{R}^{d} \rightarrow \mathbb{C}$ to $\mathbb{P}_{a}^{d}$.

Given $\omega \in \mathbb{S}_{+}^{d-1}$ and $\pi=\langle\omega\rangle^{\perp} \in \mathcal{G}_{d-1, d}$ write, for each $\xi \in \mathbb{R}^{d}, \xi=\xi^{\pi}+\xi^{\omega} \omega$, where $\xi^{\omega}=\xi \cdot \omega$. Given $s \in \mathbb{R}$ and $x \in \pi$,

$$
\begin{aligned}
\widehat{g_{j} \mathrm{~d} \sigma_{\mathbb{P} d}}(x+s \omega, t) & =\int_{\mathbb{R}^{d}} e^{i(x+s \omega) \cdot \xi+i t|\xi|^{2}} f_{j}(\xi) \mathrm{d} \xi \\
& =\int_{\pi} e^{i x \cdot \xi^{\pi}} \int_{\mathbb{R}} e^{i s \xi^{\omega}+i t\left|\xi^{\pi}\right|^{2}+i t\left|\xi^{\omega}\right|^{2}} f_{j}\left(\xi^{\pi}+\xi^{\omega} \omega\right) \mathrm{d} \xi^{\omega} \mathrm{d} \lambda_{\pi}\left(\xi^{\pi}\right) \\
& =\int_{\pi} e^{i x \cdot \xi^{\pi}} \mathcal{F}^{2}\left(g_{j, \xi^{\pi}} \mathrm{d} \sigma_{\mathbb{P}_{\left|\xi^{2}\right|^{2}}^{2}}\right)(s, t) \mathrm{d} \lambda_{\pi}\left(\xi^{\pi}\right),
\end{aligned}
$$


where $f_{j, \xi^{\pi}}(\nu):=f_{j}\left(\xi^{\pi}+\nu \omega\right), \mathcal{F}^{2}$ denotes the 2-dimensional Fourier transform and $g_{j, \xi^{\pi}}$ is the lift of $f_{j, \xi^{\pi}}$ to $\mathbb{P}_{\left|\xi^{\pi}\right|^{2}}^{2}$. Reasoning as in the proof of Theorem 1.2,

$$
\begin{aligned}
& \int_{\mathbb{R}} \int_{\mathbb{R}}\left|\partial_{s} \mathcal{R}\left(\widehat{g_{1} \mathrm{~d} \sigma_{\mathbb{P}^{d}}}(\cdot, t) \overline{\widehat{g_{2} \mathrm{~d} \sigma_{\mathbb{P}^{d}}}}(\cdot, t)\right)(\omega, s)\right|^{2} \mathrm{~d} s \mathrm{~d} t \\
& \quad=(2 \pi)^{2 d} \int_{\pi} \int_{\pi} \int_{\mathbb{R}^{4}}\left|\xi^{\omega}-\eta^{\omega} \| \zeta^{\omega}-\mu^{\omega}\right| f_{1, \xi^{\pi}}\left(\xi^{\omega}\right) \bar{f}_{2, \xi^{\pi}}\left(\eta^{\omega}\right) \bar{f}_{1, \zeta^{\pi}}\left(\mu^{\omega}\right) f_{2, \zeta^{\pi}}\left(\zeta^{\omega}\right) \mathrm{d} \Sigma_{\xi^{\pi}, \zeta^{\pi}}\left(\xi^{\omega}, \eta^{\omega}, \mu^{\omega}, \zeta^{\omega}\right)
\end{aligned}
$$

where

$\mathrm{d} \Sigma_{\xi^{\pi}, \zeta^{\pi}}\left(\xi^{\omega}, \eta^{\omega}, \mu^{\omega}, \zeta^{\omega}\right):=\delta\left(\xi^{\omega}-\eta^{\omega}+\zeta^{\omega}-\mu^{\omega}\right) \delta\left(\left(\xi^{\omega}\right)^{2}-\left(\eta^{\omega}\right)^{2}+\left(\zeta^{\omega}\right)^{2}-\left(\mu^{\omega}\right)^{2}\right) \mathrm{d} \xi^{\omega} \mathrm{d} \eta^{\omega} \mathrm{d} \mu^{\omega} \mathrm{d} \zeta^{\omega} \mathrm{d} \lambda_{\pi}\left(\xi^{\pi}\right) \mathrm{d} \lambda_{\pi}\left(\zeta^{\pi}\right)$.

Arguing similarly,

$J_{\omega}\left(\widehat{g_{1} \mathrm{~d} \sigma_{\mathbb{P} d}}, \widehat{g_{2} \mathrm{~d} \sigma_{\mathbb{P} d}}\right)=(2 \pi)^{2 d} \int_{\pi} \int_{\pi} \int_{\mathbb{R}^{4}}\left(\zeta^{\omega} \mu^{\omega}-\zeta^{\omega} \eta^{\omega}-\xi^{\omega} \mu^{\omega}+\xi^{\omega} \eta^{\omega}\right) f_{1, \xi^{\pi}}\left(\xi^{\omega}\right) \bar{f}_{2, \xi^{\pi}}\left(\eta^{\omega}\right) \bar{f}_{1, \zeta^{\pi}}\left(\mu^{\omega}\right) f_{2, \zeta^{\pi}}\left(\zeta^{\omega}\right)$

with respect to the measure $\mathrm{d} \Sigma_{\xi^{\pi}, \zeta^{\pi}}\left(\xi^{\omega}, \eta^{\omega}, \mu^{\omega}, \zeta^{\omega}\right)$, where $J_{\omega}\left(G_{1}, G_{2}\right)$ is the bilinearisation of $J_{\omega}(u)$; namely the integrand is replaced by

$$
\begin{aligned}
& G_{1}(x+s \omega, t) \partial_{s} G_{2}(y+s \omega, t)\left(\partial_{s} \bar{G}_{1}(y+s \omega, t) \bar{G}_{2}(x+s \omega, t)-\bar{G}_{1}(y+s \omega, t) \partial_{s} \bar{G}_{2}(x+s \omega, t)\right) \\
& \quad-G_{2}(y+s \omega, t) \partial_{s} G_{1}(x+s \omega, t)\left(\bar{G}_{2}(x+s \omega, t) \partial_{s} \bar{G}_{1}(y+s \omega, t)-\partial_{s} \bar{G}_{2}(x+s \omega, t) \bar{G}_{1}(y+s \omega, t)\right) .
\end{aligned}
$$

Noting that

$$
\left|\xi^{\omega}-\eta^{\omega}\right|\left|\zeta^{\omega}-\mu^{\omega}\right|+\left(\zeta^{\omega} \mu^{\omega}-\zeta^{\omega} \eta^{\omega}-\xi^{\omega} \mu^{\omega}+\xi^{\omega} \eta^{\omega}\right)=\left|\xi^{\omega}-\mu^{\omega}\right|^{2}
$$

if $\left(\xi^{\omega}, \eta^{\omega}, \mu^{\omega}, \zeta^{\omega}\right) \in \operatorname{supp}\left(\mathrm{d} \Sigma_{\xi^{\pi}, \zeta^{\pi}}\right)$, one can combine the two terms above to obtain

$$
\begin{aligned}
& \int_{\mathbb{R}} \int_{\mathbb{R}}\left|\partial_{s} \mathcal{R}\left(\widehat{g_{1} \mathrm{~d} \sigma_{\mathbb{P}^{d}}}(\cdot, t) \widehat{\widehat{g_{2} \mathrm{~d} \sigma_{\mathbb{P}}}}(\cdot, t)\right)(s, \omega)\right|^{2} \mathrm{~d} s \mathrm{~d} t+J_{\omega}\left(\widehat{g_{1} \mathrm{~d} \sigma_{\mathbb{P} d}}, \widehat{g_{2} \mathrm{~d} \sigma_{\mathbb{P}} d}\right) \\
& \quad=(2 \pi)^{2 d} \int_{\pi} \int_{\pi} \int_{\mathbb{R}^{4}}\left|\xi^{\omega}-\mu^{\omega}\right|^{2} f_{1, \xi^{\pi}}\left(\xi^{\omega}\right) \bar{f}_{2, \xi^{\pi}}\left(\eta^{\omega}\right) \bar{f}_{1, \zeta^{\pi}}\left(\mu^{\omega}\right) f_{2, \zeta^{\perp}}\left(\zeta^{\omega}\right) \mathrm{d} \Sigma_{\xi^{\pi}, \zeta^{\pi}}\left(\xi^{\omega}, \eta^{\omega}, \mu^{\omega}, \zeta^{\omega}\right) .
\end{aligned}
$$

For fixed $\xi^{\omega}$ and $\mu^{\omega}$, the only solution for the equations in the $\delta$ function is $\eta^{\omega}=\xi^{\omega}$ and $\zeta^{\omega}=\mu^{\omega}$. Thus, the right-hand side above equals

$$
\frac{(2 \pi)^{2 d}}{2} \int_{\xi^{\pi}} \int_{\zeta^{\pi}} \int_{\mathbb{R}^{2}}\left|\xi^{\omega}-\mu^{\omega}\right| f_{1, \xi^{\pi}}\left(\xi^{\omega}\right) \bar{f}_{2, \xi^{\pi}}\left(\xi^{\omega}\right) \bar{f}_{1, \zeta^{\pi}}\left(\mu^{\omega}\right) f_{2, \zeta^{\pi}}\left(\mu^{\omega}\right) \mathrm{d} \xi^{\omega} \mathrm{d} \mu^{\omega} \mathrm{d} \lambda_{\pi}\left(\xi^{\pi}\right) \mathrm{d} \lambda_{\pi}\left(\zeta^{\pi}\right)
$$

and if $f_{1}=f_{2}$,

which, of course, is

$$
\frac{(2 \pi)^{2 d}}{2} \int_{\pi} \int_{\pi} \int_{\mathbb{R}^{2}}\left|\xi^{\omega}-\mu^{\omega}\right|\left|f_{\xi^{\pi}}\left(\xi^{\omega}\right)\right|^{2}\left|f_{\zeta^{\pi}}\left(\mu^{\omega}\right)\right|^{2} \mathrm{~d} \xi^{\omega} \mathrm{d} \mu^{\omega} \mathrm{d} \lambda_{\pi}\left(\xi^{\pi}\right) \mathrm{d} \lambda_{\pi}\left(\zeta^{\pi}\right)
$$

$$
\frac{(2 \pi)^{2 d}}{2} \int_{\mathbb{R}^{d}} \int_{\mathbb{R}^{d}}|(\xi-\eta) \cdot \omega||f(\xi)|^{2}|f(\eta)|^{2} \mathrm{~d} \xi \mathrm{d} \eta .
$$

In the language of the Schrödinger equation, $u=E \widetilde{u_{0}}$, so the right-hand side is

$$
\frac{\pi}{(2 \pi)^{d+1}} \int_{\mathbb{R}^{d}} \int_{\mathbb{R}^{d}}|(\xi-\eta) \cdot \omega|\left|\widehat{u_{0}}(\xi)\right|^{2}\left|\widehat{u_{0}}(\eta)\right|^{2} \mathrm{~d} \xi \mathrm{d} \eta
$$

and one obtains the desired identity (1.10), finishing the proof of Theorem 1.1.

Remark 6.1. Averaging over all $\omega \in \mathbb{S}_{+}^{d-1}$ after dropping the term $J_{\omega}(u)$ from the obtained identity and noting that

one has

$$
\int_{\mathbb{S}^{d-1}}|(\xi-\eta) \cdot \omega| \mathrm{d} \sigma^{n}(\omega)=2|\xi-\eta| \int_{0}^{1} u\left(1-u^{2}\right)^{\frac{d-3}{2}} \mathrm{~d} u=\frac{2|\xi-\eta| \pi^{\frac{d-1}{2}}}{\Gamma((d+1) / 2)}
$$

$$
\left\|\left(-\Delta_{x}\right)^{\frac{3-d}{4}}\left(|u|^{2}\right)\right\|_{L_{x, t}^{2}\left(\mathbb{R}^{d} \times \mathbb{R}\right)}^{2} \leqslant\left.(2 \pi)^{1-d} \frac{\pi}{(2 \pi)^{d+1}} \frac{\pi^{\frac{d-1}{2}}}{\Gamma((d+1) / 2)} \int_{\mathbb{R}^{d}} \int_{\mathbb{R}^{d}}|\xi-\eta| \widehat{u_{0}}(\xi)\right|^{2}\left|\widehat{u_{0}}(\eta)\right|^{2} \mathrm{~d} \xi \mathrm{d} \eta
$$


and the constant simplifies as $\mathbf{P V}(d):=\frac{2^{-3 d} \pi \frac{1-5 d}{2}}{\Gamma\left(\frac{d+1}{2}\right)} ;$ this inequality was also obtained in $[3]$ in a more direct way.

Finally, it is noted that the honest analogue of Theorems 1.2 and 1.6 in the context of paraboloids is given by the following bilinear identity.

Theorem 6.2. Let $d \geqslant 2$ and $\omega \in \mathbb{S}_{+}^{d-1}$. Then

$$
\begin{aligned}
\int_{\mathbb{R}} \int_{\mathbb{R}}\left|\partial_{s}^{1 / 2} \mathcal{R}\left(\widehat{g_{1} \mathrm{~d} \sigma_{\mathbb{P}^{d}}}(\cdot, t) \overline{\widehat{g_{2} \mathrm{~d} \sigma_{\mathbb{P}}}}(\cdot, t)\right)(s, \omega)\right|^{2} \mathrm{~d} s \mathrm{~d} t \\
\quad=\frac{(2 \pi)^{2 d}}{2} \int_{\pi} \int_{\pi} \int_{\mathbb{R}^{2}} f_{1, \xi^{\pi}}\left(\xi^{\omega}\right) \bar{f}_{2, \xi^{\pi}}\left(\zeta^{\omega}\right) \bar{f}_{1, \zeta^{\pi}}\left(\xi^{\omega}\right) f_{2, \zeta^{\pi}}\left(\zeta^{\omega}\right) \mathrm{d} \xi^{\omega} \mathrm{d} \zeta^{\omega} \mathrm{d} \lambda_{\pi}\left(\xi^{\pi}\right) \mathrm{d} \lambda_{\pi}\left(\zeta^{\pi}\right) .
\end{aligned}
$$

The proof of Theorem 6.2 is a minor variant of the one for Theorem 1.1 exposed above: the main difference is that here one solves the equations in the $\delta$ functions in terms of $\xi^{\omega}$ and $\zeta^{\omega}$; the solution in terms of $\xi^{\omega}$ and $\mu^{\omega}$ is now degenerate in terms of the weight $\left|\xi^{\omega}-\eta^{\omega}\right|^{1 / 2}\left|\zeta^{\omega}-\mu^{\omega}\right|^{1 / 2}$, which vanishes in this case. Note that, in (6.2), the fact of taking one full derivative with respect to $s$ and adding the term $J_{\omega}\left(\widehat{g_{1} \mathrm{~d} \sigma_{\mathbb{P}^{d}}}, \widehat{g_{2} \mathrm{~d} \sigma_{\mathbb{P}^{d}}}\right)$ had the effect of replacing the weight $\left|\xi^{\omega}-\eta^{\omega} \| \zeta^{\omega}-\mu^{\omega}\right|$ by $\left|\xi^{\omega}-\mu^{\omega}\right|^{2}$ thanks to the algebraic identity (6.1), allowing one to solve in those variables.

Corollaries in the spirit of those obtained for spheres and hyperboloids can also be deduced from Theorem 6.2. In particular, the identity for complex numbers (1.12) allows one to rewrite (6.4) as the following.

Corollary 6.3. Let $d \geqslant 2$ and $\omega \in \mathbb{S}_{+}^{d-1}$. Then

$$
\begin{aligned}
\int_{\mathbb{R}} \int_{\mathbb{R}}\left|\partial_{s}^{1 / 2} \mathcal{R}\left(\widehat{g_{1} \mathrm{~d} \sigma_{\mathbb{P}} d}(\cdot, t) \widehat{\widehat{g_{2} \mathrm{~d} \sigma_{\mathbb{P}}}}(\cdot, t)\right)(s, \omega)\right|^{2} \mathrm{~d} s \mathrm{~d} t \\
\quad=\frac{(2 \pi)^{2 d}}{2} \int_{\pi} \int_{\pi} \int_{\mathbb{R}^{2}}\left|f_{1, \xi^{\pi}}\left(\xi^{\omega}\right)\right|^{2}\left|f_{2, \zeta^{\pi}}\left(\zeta^{\omega}\right)\right|^{2} \mathrm{~d} \xi^{\omega} \mathrm{d} \zeta^{\omega} \mathrm{d} \lambda_{\pi}\left(\xi^{\pi}\right) \mathrm{d} \lambda_{\pi}\left(\zeta^{\pi}\right)-I_{\omega}\left(f_{1}, f_{2}\right)
\end{aligned}
$$

where

$$
I_{\omega}\left(f_{1}, f_{2}\right):=\frac{(2 \pi)^{2 d}}{4} \int_{\pi} \int_{\pi} \int_{\mathbb{R}^{2}}\left|f_{1, \xi^{\pi}}\left(\xi^{\omega}\right) f_{2, \zeta^{\pi}}\left(\zeta^{\omega}\right)-f_{1, \zeta^{\pi}}\left(\xi^{\omega}\right) f_{2, \xi^{\pi}}\left(\zeta^{\omega}\right)\right|^{2} \mathrm{~d} \xi^{\omega} \mathrm{d} \zeta^{\omega} \mathrm{d} \lambda_{\pi}\left(\xi^{\pi}\right) \mathrm{d} \lambda_{\pi}\left(\zeta^{\pi}\right) .
$$

Note that, unlike $J_{\omega}(f)$, the term $I_{\omega}(f, f)$ does not have an obvious closed expression in terms of physical variables. Setting $f_{1}=f_{2}$ and averaging over all $\omega \in \mathbb{S}_{+}^{d-1}$ after dropping $I_{\omega}(f, f)$ one obtains

$$
\left\|\left(-\Delta_{x}\right)^{\frac{2-d}{4}}\left(|u|^{2}\right)\right\|_{L_{x, t}^{2}\left(\mathbb{R}^{d} \times \mathbb{R}\right)}^{2} \leqslant(2 \pi)^{1-d} \frac{(2 \pi)^{2 d}}{2} \frac{\left|\mathbb{S}^{d-1}\right|}{2}\left\|\widetilde{u_{0}}\right\|_{L^{2}\left(\mathbb{R}^{d}\right)}^{4}=\frac{2^{-d} \pi^{\frac{2-d}{2}}}{\Gamma(d / 2)}\left\|u_{0}\right\|_{L^{2}\left(\mathbb{R}^{d}\right)}^{4},
$$

which is the Ozawa-Tsutsumi estimate (1.8); note that for $d=2$ this amounts to the $L^{4}\left(\mathbb{R}^{2+1}\right)$ Strichartz estimate. The interested reader should look at the work of Bennett, Bez, Jeavons and Pattakos [3] for a unified treatment of the Ozawa-Tsutsumi estimates (1.8), the inequalities deduced from (6.3), and a more general case with an arbitrary number of derivatives on the lefthand-side of such inequalities.

\section{REFERENCES}

[1] J. Bennett. Aspects of multilinear harmonic analysis related to transversality. In Harmonic analysis and partial differential equations, volume 612 of Contemp. Math., pages 1-28. Amer. Math. Soc., Providence, RI, 2014.

[2] J. Bennett, N. Bez, T. C. Flock, S. Gutiérrez, and M. Iliopoulou. A sharp $k$-plane Strichartz inequality for the Schrödinger equation. Trans. Amer. Math. Soc., 370(8):5617-5633, 2018.

[3] J. Bennett, N. Bez, C. Jeavons, and N. Pattakos. On sharp bilinear Strichartz estimates of Ozawa-Tsutsumi type. J. Math. Soc. Japan, 69(2):459-476, 2017.

[4] J. Bennett, A. Carbery, and T. Tao. On the multilinear restriction and Kakeya conjectures. Acta Math., 196(2):261-302, 2006.

[5] J. Bennett and M. Iliopoulou. A multilinear Fourier extension identity on $\mathbb{R}^{n}$. Math. Res. Lett., 25(4):1089$1108,2018$. 
[6] J. Bennett and S. Nakamura. Tomography bounds for the Fourier extension operator. In preparation.

[7] N. Bez and K. M. Rogers. A sharp Strichartz estimate for the wave equation with data in the energy space. J. Eur. Math. Soc. (JEMS), 15(3):805-823, 2013.

[8] J. Bourgain. Refinements of Strichartz' inequality and applications to 2D-NLS with critical nonlinearity. Internat. Math. Res. Notices, (5):253-283, 1998.

[9] J. Bourgain. Global wellposedness of defocusing critical nonlinear Schrödinger equation in the radial case. J. Amer. Math. Soc., 12(1):145-171, 1999.

[10] E. Carneiro. A sharp inequality for the Strichartz norm. Int. Math. Res. Not. IMRN, (16):3127-3145, 2009.

[11] E. Carneiro and D. Oliveira e Silva. Some sharp restriction inequalities on the sphere. Int. Math. Res. Not. IMRN, (17):8233-8267, 2015.

[12] E. Carneiro, D. Oliveira e Silva, and M. Sousa. Extremizers for Fourier restriction on hyperboloids. Ann. Inst. H. Poincaré Anal. Non Linéaire, 36(2):389-415, 2019.

[13] M. Christ and S. Shao. Existence of extremals for a Fourier restriction inequality. Anal. PDE, 5(2):261-312, 2012.

[14] M. Christ and S. Shao. On the extremizers of an adjoint Fourier restriction inequality. Adv. Math., 230(3):957977, 2012.

[15] J. Colliander, M. Grillakis, and N. Tzirakis. Tensor products and correlation estimates with applications to nonlinear Schrödinger equations. Comm. Pure Appl. Math., 62(7):920-968, 2009

[16] J. Colliander, M. Keel, G. Staffilani, H. Takaoka, and T. Tao. Global well-posedness and scattering for the energy-critical nonlinear Schrödinger equation in $\mathbb{R}^{3}$. Ann. of Math. (2), 167(3):767-865, 2008.

[17] C. Fefferman. Inequalities for strongly singular convolution operators. Acta Math., 124:9-36, 1970.

[18] D. Foschi. Maximizers for the Strichartz inequality. J. Eur. Math. Soc. (JEMS), 9(4):739-774, 2007.

[19] D. Foschi. Global maximizers for the sphere adjoint Fourier restriction inequality. J. Funct. Anal., 268(3):690$702,2015$.

[20] D. Foschi and S. Klainerman. Bilinear space-time estimates for homogeneous wave equations. Ann. Sci. École Norm. Sup. (4), 33(2):211-274, 2000.

[21] D. Foschi and D. Oliveira e Silva. Some recent progress on sharp Fourier restriction theory. Anal. Math., 43(2):241-265, 2017.

[22] S. Helgason. The Radon transform, volume 5 of Progress in Mathematics. Birkhäuser Boston, Inc., Boston, MA, second edition, 1999.

[23] C. Jeavons. A sharp bilinear estimate for the Klein-Gordon equation in arbitrary space-time dimensions. Differential Integral Equations, 27(1-2):137-156, 2014.

[24] J. E. Lin and W. A. Strauss. Decay and scattering of solutions of a nonlinear Schrödinger equation. J. Functional Analysis, 30(2):245-263, 1978.

[25] C. S. Morawetz. Time decay for the nonlinear Klein-Gordon equations. Proc. Roy. Soc. London Ser. A, 306:291$296,1968$.

[26] K. Nakanishi. Energy scattering for nonlinear Klein-Gordon and Schrödinger equations in spatial dimensions 1 and 2. J. Funct. Anal., 169(1):201-225, 1999.

[27] T. Ozawa and K. M. Rogers. A sharp bilinear estimate for the Klein-Gordon equation in $\mathbb{R}^{1+1}$. Int. Math. Res. Not. IMRN, (5):1367-1378, 2014.

[28] T. Ozawa and Y. Tsutsumi. Space-time estimates for null gauge forms and nonlinear Schrödinger equations. Differential Integral Equations, 11(2):201-222, 1998.

[29] F. Planchon and L. Vega. Bilinear virial identities and applications. Ann. Sci. Éc. Norm. Supér. (4), 42(2):261290, 2009.

[30] R. Quilodrán. Nonexistence of extremals for the adjoint restriction inequality on the hyperboloid. J. Anal. Math., 125:37-70, 2015.

[31] R. S. Strichartz. Restrictions of Fourier transforms to quadratic surfaces and decay of solutions of wave equations. Duke Math. J., 44(3):705-714, 1977.

[32] T. Tao. A sharp bilinear restrictions estimate for paraboloids. Geom. Funct. Anal., 13(6):1359-1384, 2003.

[33] T. Tao. Some recent progress on the restriction conjecture. In Fourier analysis and convexity, Appl. Numer. Harmon. Anal., pages 217-243. Birkhäuser Boston, Boston, MA, 2004.

[34] P. A. Tomas. A restriction theorem for the Fourier transform. Bull. Amer. Math. Soc., 81:477-478, 1975.

[35] T. Wolff. A sharp bilinear cone restriction estimate. Ann. of Math. (2), 153(3):661-698, 2001.

[36] A. Zygmund. On Fourier coefficients and transforms of functions of two variables. Studia Math., 50:189-201, 1974 .

David Beltran: Department of Mathematics, University of Wisconsin, 480 Lincoln Drive, Madison, WI, 53706, USA.

E-mail address: dbeltran@math.wisc.edu 
Luis Vega: Departamento de Matematicas, Universidad del Pais Vasco/Euskal Herriko Unibertsitatea (UpV/EhU), Aptdo. 644, 48080, Bilbao, Spain, and Basque Center for Applied Mathematics (BCAM), Alameda de Mazarredo 14, 48009, Bilbao, Spain

E-mail address: lvega@bcamath.org, luis.vega@ehu.es 